\title{
Mass dependence of Higgs boson production at large transverse momentum through a bottom-quark loop
}

\author{
Eric Braaten, ${ }^{1, *}$ Hong Zhang, ${ }^{1, \dagger}$ and Jia-Wei Zhang ${ }^{2,+}$ \\ ${ }^{1}$ Department of Physics, The Ohio State University, Columbus, Ohio 43210, USA \\ ${ }^{2}$ Department of Physics, Chongqing University of Science and Technology, Chongqing 401331, China
}

(Received 21 August 2017; published 29 May 2018)

\begin{abstract}
In the production of the Higgs through a bottom-quark loop, the transverse momentum distribution of the Higgs at large $P_{T}$ is complicated by its dependence on two other important scales: the bottom quark mass $m_{b}$ and the Higgs mass $m_{H}$. A strategy for simplifying the calculation of the cross section at large $P_{T}$ is to calculate only the leading terms in its expansion in $m_{b}^{2} / P_{T}^{2}$. In this paper, we consider the bottom-quark-loop contribution to the parton process $q \bar{q} \rightarrow H+g$ at leading order in $\alpha_{s}$. We show that the leading power of $1 / P_{T}^{2}$ can be expressed in the form of a factorization formula that separates the large scale $P_{T}$ from the scale of the masses. All the dependence on $m_{b}$ and $m_{H}$ can be factorized into a distribution amplitude for $b \bar{b}$ in the Higgs, a distribution amplitude for $b \bar{b}$ in a real gluon, and an end point contribution. The factorization formula can be used to organize the calculation of the leading terms in the expansion in $m_{b}^{2} / P_{T}^{2}$ so that every calculation involves at most two scales.
\end{abstract}

DOI: 10.1103/PhysRevD.97.096014

\section{INTRODUCTION}

The discovery of the Higgs boson in the year 2012 completed the Standard Model (SM) of particle physics [1,2]. Many properties of the Higgs have since been measured, and they are in agreement with the theoretical predictions of the SM [3]. As the experimental precision improves with the collection of more and more data at the Large Hadron Collider (LHC), it is important that theoretical uncertainties in the SM predictions are under control. The most straightforward way to reduce the theoretical uncertainties is to carry out calculations to higher orders in perturbation theory and to resum to all orders logarithmic terms that spoil the perturbative expansion in certain kinematic regions.

The dominant contribution to the cross section for Higgs production is through a top-quark loop due to the large Yukawa coupling $y_{t}=m_{t} / v$ of the top quark, where $m_{t}$ is the top-quark mass and $v=246 \mathrm{GeV}$ is the vacuum expectation value of the Higgs field. The contribution to the cross section from the interference between the bottomquark loop and the top-quark loop can be as large as $8 \%$ of

\footnotetext{
*braaten@mps.ohio-state.edu tzhang.5676@osu.edu

jwzhang@cqust.edu.cn
}

Published by the American Physical Society under the terms of the Creative Commons Attribution 4.0 International license. Further distribution of this work must maintain attribution to the author(s) and the published article's title, journal citation, and DOI. Funded by SCOAP. the contribution from the top-quark loop [4]. That contribution is suppressed by the Yukawa coupling $y_{b}=m_{b} / v$, where $m_{b}$ is the bottom-quark mass. However, the amplitude for the parton process $i j \rightarrow H+k$ from a bottomquark loop at leading order (LO) in the QCD coupling constant $\alpha_{s}$ reveals the existence of double logarithms of $m_{H}^{2} / m_{b}^{2}$ and $P_{T}^{2} / m_{b}^{2}$, where $m_{H}$ is the mass of the Higgs and $P_{T}$ is its transverse momentum. Since the high-lminosity LHC may be able to measure the Higgs $P_{T}$ distribution with a few percent accuracy, it is important to understand the bottom-quark-loop contribution to the same level of precision. This requires calculating the process to higher orders in $\alpha_{s}$ and resumming large logarithms to all orders.

The $b$-quark-loop contribution to the Higgs $P_{T}$ distribution was first calculated in 1987 [5,6], but a complete calculation at next-to-leading order (NLO) in $\alpha_{s}$ is still not available. The difficulty of the NLO calculation is mainly due to the existence of multiple scales, including the two mass scales $m_{H}$ and $m_{b}$ and the two kinematic scales $P_{T}$ and $\sqrt{\hat{s}}$, where $\hat{s}$ is the square of the parton center-of-mass energy. In the kinematic region where $m_{b}$ is much smaller than the other scales, the calculation can be simplified by taking the limit $m_{b} \rightarrow 0$. This limit is nontrivial because of nonanalytic functions of $m_{b}$, such as $\log \left(P_{T}^{2} / m_{b}^{2}\right)$, that diverge in the $m_{b} \rightarrow 0$ limit. The $m_{b} \rightarrow 0$ limit can be calculated by solving the differential equations for master integrals in this limit [7]. This method has been used by Mueller and Öztürk to calculate bottom-quark-loop contributions to the inclusive cross section for Higgs production to NLO [7]. The method has also been used by 
Melnikov, Tancredi, and Wever to calculate the helicity amplitudes for $i j \rightarrow H+k$ from a bottom-quark loop at NLO [8,9]. Terms suppressed by powers of $m_{b}$ are neglected, and all logarithms of $m_{b}$ are included. As discussed in Ref. [4], calculating the limit $m_{b} \rightarrow 0$ using differential equations for master integrals is very demanding of computing resources. A method for taking the limit at an earlier stage of the calculation would be desirable.

An NLO calculation does not necessarily produce a dramatic increase in accuracy. The relative error in an exclusive amplitude is probably order $\alpha_{s}^{2} \log ^{4}\left(P_{T} / m_{b}\right)$, and the relative error in a sufficiently inclusive cross section is order $\alpha_{s}^{2} \log ^{2}\left(P_{T} / m_{b}\right)$. To decrease the relative error to order $\alpha_{s}$ requires resumming the leading logarithms to all orders. Much effort has been devoted to resumming the large logarithms of $P_{T} / m_{b}$ with $P_{T} \ll m_{b}$ [10-20]. The sources of large logarithms of $m_{b} / P_{T}$ with $P_{T} \gg m_{b}$ are completely different. At leading order, the large logarithms come from collinear regions of the loop momentum in which $b$ and $\bar{b}$ have nearly collinear momenta and from soft regions in which $b$ or $\bar{b}$ has momentum small compared to $P_{T}$. The effects of partial resummation of large logarithms of $m_{b} / P_{T}$ have been studied empirically without understanding their origin at higher orders [21-24]. The resummation of logarithms in abelian QCD has been studied in Ref. [25]. It would be useful to develop systematic methods to resum large logarithms from QCD radiative corrections.

Calculations to higher orders can be simplified, and the resummation of logarithms can be facilitated by separating scales. An example is the Higgs effective field theory (HEFT), in which the top-quark mass $m_{t}$ is taken to be much larger than all other scales and the top quark is integrated out of the theory. Using HEFT, the total cross section for Higgs production has been calculated to the impressive precision of $\mathrm{N}^{3} \mathrm{LO}[26,27]$. The accuracy has been further improved by the resummation of threshold logarithms [28-33]. HEFT has also been used to calculate the cross section for Higgs plus one jet to $\mathrm{N}^{2} \mathrm{LO}$ [34-37] and the cross section for Higgs plus two or more jets to NLO [38-40].

A new approach to Higgs production at large transverse momentum $P_{T}$ based on separation of scales has been introduced in Refs. [41,42]. The separation of scales was accomplished by using factorization formulas that were deduced from factorization theorems for perturbative QCD. When there is a large kinematic scale $Q$, it is reasonable to expand in powers of $M^{2} / Q^{2}$, where $M$ represents scales provided by masses and nonperturbative low-energy scales. The expansion may not be straightforward because of terms that are nonanalytic in $M^{2} / Q^{2}$, such as logarithms of $M^{2}$ or functions of mass ratios. In Ref. [41], a factorization formula for the inclusive Higgs $P_{T}$ distribution at the leading power of $M^{2} / P_{T}^{2}$ was used to factor the nonanalytic terms into fragmentation functions. The factorization formula reproduces the $\mathrm{LO}$ result up to corrections of order $M^{2} / Q^{2}$, indicating that theoretical errors are under control at large $Q^{2}$. In the factorization approach, different energy scales are separated into different pieces in the factorization formula. Since fewer scales need to be considered in each piece, calculations to higher order are much simpler. The factorization formula also makes it possible to sum large logarithms of $M^{2} / P_{T}^{2}$ to all orders by solving evolution equations for the fragmentation functions.

In Ref. [42], we showed that the factorization approach can also be used to simplify amplitudes for Higgs production through a top quark loop at large $P_{T}$. The all-orders proof of factorization and the renormalization group equations are very similar to those for the inclusive production of heavy quarkonium $[43,44]$. The specific example considered in Ref. [42] was the top-quark-loop contribution to the parton process $q \bar{q} \rightarrow H+g$ at LO. The relevant scales are the hard kinematic scales $Q \sim P_{T}, \sqrt{\hat{s}}$ and the soft mass scales $M \sim m_{H}, m_{t}$. The leading power in the expansion of the amplitude in powers of $M^{2} / Q^{2}$ was expressed in the form of a factorization formula in which the scales $M$ and $Q$ are separated. The factorization formula involves a distribution amplitude for a $t \bar{t}$ pair in the Higgs, a distribution amplitude for a $t \bar{t}$ pair in a real gluon, and an end point contribution from the transition $t+\bar{t} \rightarrow H+g$ via the exchange of a soft quark. The factorization formula provides a systematic approximation with errors of order $M^{2} / Q^{2}$ that go to zero as the kinematic scale $Q$ increases. Every piece in the factorization formula was calculated diagrammatically in such a way that it involved either the scale $Q$ or the scale $M$. We also presented an improved factorization formula that includes all dependence on $m_{t}$ that is not suppressed by $m_{H}^{2} / Q^{2}$, so that the largest errors are reduced from order $m_{t}^{2} / Q^{2}$ to order $m_{H}^{2} / Q^{2}$.

In this paper, we apply the factorization method of Ref. [42] to the bottom-quark-loop contribution to the parton process $q \bar{q} \rightarrow H+g$ at LO. The relevant scales are the hard kinematic scales $Q \sim P_{T}, \sqrt{\hat{s}}$ and the soft mass scales $M \sim m_{H}, m_{b}$. The leading power in the expansion of the amplitude in powers of $M^{2} / Q^{2}$ is expressed in the form of a factorization formula in which the scales $M$ and $Q$ are separated. We also present an improved factorization formula that includes all dependence on $m_{H}$ that is not suppressed by $m_{b}^{2} / Q^{2}$, so that the largest errors are reduced from order $m_{H}^{2} / Q^{2}$ to order $m_{b}^{2} / Q^{2}$.

This paper is organized as follows. In Sec. II, we introduce the form factor for the bottom-quark-loop contribution to the matrix element for $q \bar{q} \rightarrow H+g$. We define the leading-power (LP) form factor to be the leading term in the expansion of the form factor in powers of $M^{2} / Q^{2}$. In Sec. III, we calculate the LP form factor using dimensional regularization and rapidity regularization to regularize the divergences that arise from separating the contributions from different regions. In Sec. IV, we renormalize all the ultraviolet divergences to obtain a finite factorization formula for the LP form factor. We also present an improved factorization formula with errors of order $m_{b}^{2} / Q^{2}$. In Sec. V, we show that the improved factorization 
formula gives a good approximation to the full form factor whose error decreases to 0 rapidly as $P_{T}$ increases. We discuss the prospects for extending our approach to NLO in $\alpha_{s}$ in Sec. VI.

\section{HIGGS PRODUCTION BY $q \bar{q} \rightarrow H+g$ THROUGH A $b$ LOOP}

In this section, we define the form factor that determines the bottom-quark-loop contribution to the cross section for $q \bar{q} \rightarrow H+g$ at leading order in $\alpha_{s}$. We give the leading power in the expansion of the form factor in powers of $M^{2} / Q^{2}$. We also present the schematic form of a factorization formula for the LP form factor.

\section{A. Form factor for $\boldsymbol{g}^{*} \rightarrow \boldsymbol{H}+\boldsymbol{g}$}

The reaction $q \bar{q} \rightarrow H+g$ proceeds at leading order in the QCD coupling constant $g_{s}$ through the two one-loop Feynman diagrams in Fig. 1. The matrix element for $q\left(p_{1}\right) \bar{q}\left(p_{2}\right) \rightarrow H(P)+g\left(p_{3}\right)$ at $\mathrm{LO}$ has the form
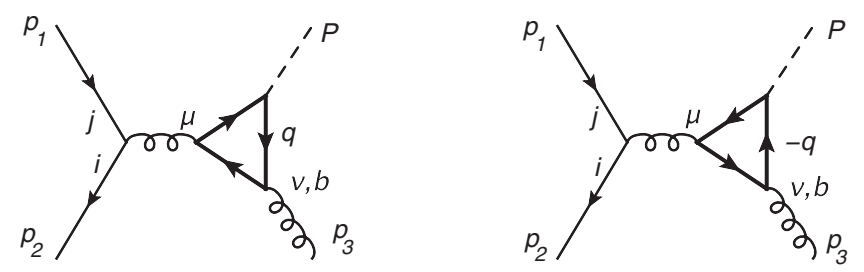

FIG. 1. Feynman diagrams for $q \bar{q} \rightarrow H+g$ through a bottomquark loop at LO.

$$
\mathcal{M}=\frac{g_{s}}{2 \hat{s}} T_{i j}^{b} \bar{v}_{2} \gamma_{\mu} u_{1} \mathcal{T}^{\mu \nu}\left(P, p_{3}\right) \varepsilon_{3 \nu}^{*}
$$

where $T_{i j}^{b}$ is the color factor, $\bar{v}_{2}$ and $u_{1}$ are the Dirac spinors for $\bar{q}$ and $q$, and $\varepsilon_{3}$ is the polarization vector for the finalstate gluon. The $q \bar{q}$ invariant mass $\hat{s}=\left(p_{1}+p_{2}\right)^{2}$ is also the invariant mass of the Higgs and the final-state gluon. The bottom-quark-loop contribution to the amplitude $\mathcal{T}^{\mu \nu}$ for $g^{*} \rightarrow H+g$ is

$$
\mathcal{T}^{\mu \nu}\left(P, p_{3}\right)=i g_{s}^{2} y_{b} \int_{q} \frac{\operatorname{Tr}\left[\left(\not{q}+\not P+m_{b}\right) \gamma^{\mu}\left(\not q-\not \not_{3}+m_{b}\right) \gamma^{\nu}\left(\not q+m_{b}\right)\right]-\left(m_{b} \rightarrow-m_{b}\right)}{\left[(q+P)^{2}-m_{b}^{2}+i \epsilon\right]\left[q^{2}-m_{b}^{2}+i \epsilon\right]\left[\left(q-p_{3}\right)^{2}-m_{b}^{2}+i \epsilon\right]},
$$

where the integration measure is $\int_{q}=\int d^{4} q /(2 \pi)^{4}$. The color trace $\operatorname{tr}\left(T^{a} T^{b}\right)$ has been absorbed into the prefactor of $\mathcal{T}^{\mu \nu}$ in Eq. (1). The explicit Dirac trace in Eq. (2) comes from the first diagram in Fig. 1. Since the only nonzero terms in the trace are proportional to $m_{b}$ or $m_{b}^{3}$, the two diagrams are equal.

The Ward identities $\left(P+p_{3}\right)_{\mu} \mathcal{T}^{\mu \nu}=0$ and $p_{3 \nu} \mathcal{T}^{\mu \nu}=0$ imply that the tensor $\mathcal{T}^{\mu \nu}$ can be expressed in terms of two scalar form factors that are dimensionless functions of $\hat{s}$ and masses [45-47]. Only one of the form factors contributes to the matrix element $\mathcal{M}$ in Eq. (1). It can be expressed as

$$
\mathcal{F}\left(\hat{s}, m_{b}^{2}, m_{H}^{2}\right)=\frac{1}{(D-2) 4 m_{b}}\left(g_{\mu \nu}-\frac{p_{3 \mu}\left(P+p_{3}\right)_{\nu}}{P \cdot p_{3}}\right) \mathcal{T}^{\mu \nu}\left(P, p_{3}\right),
$$

where $D=4$ is the number of space-time dimensions. The contribution to the form factor from a bottom-quark loop can be expressed as an integral over a loop momentum:

$$
\mathcal{F}\left(\hat{s}, m_{b}^{2}, m_{H}^{2}\right)=i g_{s}^{2} y_{b} \int_{q} \frac{q^{2}+2 p_{3} \cdot q+2 P \cdot p_{3}+3 m_{b}^{2}-4\left(P+p_{3}\right) \cdot q p_{3} \cdot q / P \cdot p_{3}}{\left[(q+P)^{2}-m_{b}^{2}+i \epsilon\right]\left[q^{2}-m_{b}^{2}+i \epsilon\right]\left[\left(q-p_{3}\right)^{2}-m_{b}^{2}+i \epsilon\right]} .
$$

The square of the matrix element $\mathcal{M}$ for $q \bar{q} \rightarrow H+g$ summed over spins and colors is proportional to $|\mathcal{F}|^{2}$ :

$$
\frac{1}{4 N_{c}^{2}} \sum|\mathcal{M}|^{2}=\frac{2\left(N_{c}^{2}-1\right) g_{s}^{2} m_{b}^{2}}{N_{c}^{2}} \frac{\hat{t}^{2}+\hat{u}^{2}}{\hat{s}\left(\hat{s}-m_{H}^{2}\right)^{2}}\left|\mathcal{F}\left(\hat{s}, m_{b}^{2}, m_{H}^{2}\right)\right|^{2} .
$$

The bottom-quark-loop contribution to the matrix elements for $g q \rightarrow H+q$ and $g \bar{q} \rightarrow H+\bar{q}$ at LO can be expressed in terms of the same function $\mathcal{F}$ as the form factor for $q \bar{q} \rightarrow H+g$ but with the positive Mandelstam variable $\hat{s}$ replaced by a negative Mandelstam variable $\hat{t}$. If the form factor $\mathcal{F}$ for $q \bar{q} \rightarrow H+g$ is expressed in terms of the complex variable $\hat{s}+i \epsilon$, it can be applied to $g q \rightarrow H+q$ and $g \bar{q} \rightarrow H+\bar{q}$ by analytic continuation.

\section{B. LP form factor}

The form factor $\mathcal{F}$ is a function of the three energy scales $\hat{s}^{1 / 2}, m_{b}$, and $m_{H}$, which satisfy the inequalities $m_{b}<m_{H} \leq \sqrt{\hat{s}}$. Analytic expressions for $\mathcal{F}$ are given in Refs. [5,6]. The analytic expression for $\mathcal{F}$ can be simplified in the limit $m_{b}, m_{H} \ll \hat{s}^{1 / 2}$ by expanding in powers of $m_{H}^{2} / \hat{s}$ and $m_{b}^{2} / \hat{s}$. We refer to the leading term in the expansion of the form factor in powers of $m_{b}^{2} / \hat{s}$ and $m_{H}^{2} / \hat{s}$ as the leading-power form factor. The LP form factor can be derived from the full form factor in Refs. [5,6]:

\footnotetext{
${ }^{1}$ We treat the dimensionless Yukawa coupling $y_{b}$ as an independent parameter from the quark mass $m_{b}$.
} 
$\mathcal{F}^{\mathrm{LP}}\left(\hat{s}, m_{b}^{2}, m_{H}^{2}\right)=\frac{g_{s}^{2} y_{b}}{32 \pi^{2}}\left\{-\log ^{2} \frac{-\hat{s}-i \epsilon}{m_{b}^{2}}+4 \log \frac{-\hat{s}-i \epsilon}{m_{b}^{2}}+\left(\log \frac{r+\sqrt{r^{2}-1}}{r-\sqrt{r^{2}-1}}-i \pi\right)^{2}-\frac{4 \sqrt{r^{2}-1}}{r}\left(\log \frac{r+\sqrt{r^{2}-1}}{r-\sqrt{r^{2}-1}}-i \pi\right)-4\right\}$,

where $r$ is the mass ratio defined by

$$
r \equiv m_{H} /\left(2 m_{b}\right)
$$

It can also be obtained from the top-quark loop contribution to the LP form factor in Ref. [42] by analytically continuing the topquark mass $m_{t}$ to $m_{b}-i \epsilon$ and replacing the top-quark Yukawa coupling $y_{t}$ by $y_{b}$.

Another limit in which the analytic expression for $\mathcal{F}$ can be simplified is $m_{b} \ll m_{H}, \hat{s}^{1 / 2}$. The leading term in the expansion in powers of $m_{b}^{2} / m_{H}^{2}$ and $m_{b}^{2} / \hat{s}$ depends logarithmically on $m_{b}$, and we must keep the $m_{b}$ dependence in the logarithms. The $m_{b} \rightarrow 0$ limit of the form factor is

$$
\mathcal{F}\left(\hat{s}, m_{b}^{2} \rightarrow 0, m_{H}^{2}\right)=\frac{g_{s}^{2} y_{b}}{32 \pi^{2}}\left\{-\log ^{2} \frac{-\hat{s}-i \epsilon}{m_{H}^{2}}-\left(2 \log \frac{m_{H}^{2}}{m_{b}^{2}}-\frac{4 \hat{s}}{\hat{s}-m_{H}^{2}}\right) \log \frac{\hat{s}+i \epsilon}{m_{H}^{2}}-\pi^{2}-4\right\} .
$$

\section{Leading-power regions}

The LP form factor in Eq. (6) can be calculated directly using the method of regions $[48,49]$. There are four regions of the loop integral over the momentum $q$ in Eq. (4) that contribute at leading power:

(i) the hard region, in which $q^{\mu}$ is order $Q$, so $q^{2}, P . q$, and $p_{3} . q$ are all order $Q^{2}$,

(ii) the Higgs collinear region, in which $p_{3} . q$ is order $Q^{2}$, but $q^{2}$ and P.q are order $M^{2}$,

(iii) the gluon collinear region, in which $P . q$ is order $Q^{2}$, and $q^{2}$ and $p_{3} . q$ are order $M^{2}$, and (iv) the soft region, in which $q^{\mu}$ is order $M$, so $q^{2}$ is order $M^{2}$, and $P . q$ and $p_{3} . q$ are order $M Q$.

The LP form factor $\mathcal{F}$ in Eq. (6) is finite, but the contributions from the individual leading-power regions have ultraviolet divergences and infrared divergences. The divergences cancel when all the contributions are added. Some of the divergences can be regularized using dimensional regularization. The generalization of the integral in Eq. (4) to $D=$ $4-2 \epsilon$ space-time dimensions can be obtained by the contraction in Eq. (3) of the tensor $\mathcal{T}^{\mu \nu}$ in Eq. (2). After evaluating the Dirac trace, the form factor reduces to

$$
\begin{aligned}
\mathcal{F}\left(\hat{s}, m_{b}^{2}, m_{H}^{2}\right)= & \frac{2 i g_{s}^{2} y_{b}}{D-2} \int_{q} \frac{1}{\left[(q+P)^{2}-m_{b}^{2}+i \epsilon\right]\left[q^{2}-m_{b}^{2}+i \epsilon\right]\left[\left(q-p_{3}\right)^{2}-m_{b}^{2}+i \epsilon\right]} \\
& \times\left((5-D) q^{2}-4 \frac{\left(P+p_{3}\right) \cdot q p_{3} \cdot q}{P \cdot p_{3}}+2(D-3) p_{3} \cdot q+(D-2) P \cdot p_{3}+(D-1) m_{b}^{2}\right),
\end{aligned}
$$

where the integration measure for the loop momentum is

$$
\int_{q} \equiv \mu^{2 \epsilon} \frac{(4 \pi)^{-\epsilon}}{\Gamma(1+\epsilon)} \int \frac{d^{D} q}{(2 \pi)^{D}}
$$

The regularized contributions to the LP form factor from each of the regions itemized above can be obtained from the expression for the integral in Eq. (9) by keeping only the leading terms at large $Q / M$ in the numerator and the leading terms in each of the denominators.

\section{Factorization formula}

In order to understand the dependence of the leadingpower form factor in Eq. (6) on the masses, it is necessary to separate the dependence on $\hat{s}$ from the dependence on the masses $m_{H}$ and $m_{b}$. We refer to the kinematic scale $Q=\hat{s}^{1 / 2}$ as the hard scale. We refer to the scale $M$ provided by the masses $m_{H}$ and $m_{b}$ as the soft scale. The four regions itemized above correspond to four contributions to the LP form factor:

(i) direct production of $H+g$, in which the Higgs $H$ and the real gluon $g$ are produced by the process $g^{*} \rightarrow H+g$ at the hard scale $Q$,

(ii) $b \bar{b}$ fragmentation into $H$, in which a nearly collinear $b \bar{b}$ pair and the real gluon are created by the process $g^{*} \rightarrow b \bar{b}+g$ at the hard scale $Q$, and the Higgs is produced by the subsequent transition $b \bar{b} \rightarrow H$ at the soft scale $M$,

(iii) $b \bar{b}$ fragmentation into $g$, in which a nearly collinear $b \bar{b}$ pair and the Higgs are created by the process $g^{*} \rightarrow H+b \bar{b}$ at the hard scale $Q$, and the real gluon is produced by the subsequent transition $b \bar{b} \rightarrow g$ at the soft scale $M$, 
(iv) end point production of $H+g$, in which a $b$ and $\bar{b}$ are created by the process $g^{*} \rightarrow b+\bar{b}$ at the hard scale $Q$, and the Higgs and the real gluon are produced by the subsequent transition $b+\bar{b} \rightarrow H+$ $g$ at the soft scale $M$.

In Ref. [42], we showed that the top-quark-loop contribution to the LP form factor for $g^{*} \rightarrow H+g$ at LO can be expressed in terms of a factorization formula that separates the hard scale $Q$ from the masses $m_{t}$ and $m_{H}$. The analogous factorization formula for the bottomquark-loop contribution to the LP form factor has the schematic form

$$
\begin{aligned}
\mathcal{F}^{\mathrm{LP}}[H+g]= & \tilde{\mathcal{F}}[H+g]+\tilde{\mathcal{F}}\left[b \bar{b}_{1 V}+g\right] \otimes d\left[b \bar{b}_{1 V} \rightarrow H\right] \\
& +\tilde{\mathcal{F}}\left[H+b \bar{b}_{8 T}\right] \otimes d\left[b \bar{b}_{8 T} \rightarrow g\right] \\
& +\mathcal{F}_{\text {endpt }}[H+g] .
\end{aligned}
$$

The terms on the right side correspond to the four contributions itemized above. The subscripts on $b \bar{b}$ indicate the color channel, which can be color-singlet (1) or coloroctet (8), and the Lorentz channel, which can be vector $(V)$ or tensor $(T)$. The $\otimes$ represents an integral over the relative longitudinal momentum fraction $\zeta$ of the $b \bar{b}$ pair, whose range is $-1 \leq \zeta \leq+1$. The factors represented by $\tilde{\mathcal{F}}$ are hard form factors that depend only on the hard scale $Q$. The factors represented by $d$ are distribution amplitudes that depend only on the soft scale $M$. Regularized expressions for each of the pieces in the factorization formula in Eq. (11) will be obtained in Sec. III. Renormalized expressions for each of the pieces in the factorization formula will be given in Sec. IV.

\section{REGULARIZED FACTORIZATION FORMULA}

In this section, we calculate each of the pieces in the factorization formula for the LP form factor in Eq. (11) in a way that involves only the single scale $Q$ or $M$. We use dimensional regularization and rapidity regularization to regularize the divergences in the contributions to the LP form factor from each of the leading-power regions. The divergences cancel when the four terms on the right side of Eq. (11) are added.

\section{A. Rapidity regularization and zero-bin subtraction}

The contributions to the LP form factor from the individual leading-power regions have ultraviolet divergences and infrared divergences. Some of the divergences are regularized by the dimensional regularization of the loop integral in Eq. (9). There are additional infrared divergences called rapidity divergences that require some other regularization procedure. We regularize the rapidity divergences using a method called rapidity regularization. Rapidity regularization in conjunction with zero-bin subtraction was introduced as a method for regularizing rapidity divergences by Manohar and Stewart [50]. Rapidity regularization separates the contributions from collinear and soft regions by explicitly breaking the boost invariance. Zero-bin subtractions of collinear contributions are required to avoid double counting of soft contributions. With rapidity regularization and zero-bin subtraction, the rapidity divergence from each region is an ultraviolet divergence. This allows the cancellation of rapidity divergences to be implemented as a renormalization procedure.

In order to specify the rapidity regularization factors, it is convenient to introduce light-like vectors $n$ and $\bar{n}$ such that the only components of $P^{\mu}$ and $p_{3}^{\mu}$ that are of order $Q$ are $P . n$ and $p_{3} . \bar{n}$. We choose the normalizations of $n$ and $\bar{n}$ so that $n \cdot \bar{n}=2$, which implies $P . n p_{3} \cdot \bar{n}=\hat{s}$. Dimensional regularization is used to separate the hard contribution from the sum of the remaining contributions. The integration measure of the loop momentum in Eq. (10) can be expressed as

$$
\int_{q} \equiv \int \frac{d(q \cdot n) d(q \cdot \bar{n})}{8 \pi^{2}} \int_{q_{\perp}},
$$

where the measure of the dimensionally regularized transverse momentum integral is

$$
\int_{\boldsymbol{q}_{\perp}} \equiv \mu^{2 \epsilon} \frac{(4 \pi)^{-\epsilon}}{\Gamma(1+\epsilon)} \int \frac{d^{2-2 \epsilon} q_{\perp}}{(2 \pi)^{2-2 \epsilon}} .
$$

We can use the 4-vectors $n$ and $\bar{n}$ to define regions of the loop momentum $q$. In the $n$ collinear region, $q . \bar{n}$ is order $Q$, $q^{2}$ is order $M^{2}$, and $q . n$ is order $M^{2} / Q$. In the $\bar{n}$ collinear region, $q . n$ is order $Q, q^{2}$ is order $M^{2}$, and $q . \bar{n}$ is order $M^{2} / Q$. In the soft region, q.n, $q . \bar{n}$, and $\boldsymbol{q}_{\perp}$ are all order $M$.

With rapidity regularization, different regularization factors may be used in different regions. The specific forms of the regularization factors required for our problem were used in Ref. [51] and described more explicitly in Ref. [52]. The regularization factors in each of the regions of $q$ are

$$
\begin{aligned}
& n \text { collinear: }\left(|q \cdot \bar{n}| / \nu_{-}\right)^{-\eta}, \\
& \bar{n} \text { collinear: }\left(|q \cdot n| / \nu_{+}\right)^{-\eta}, \\
& \text { soft: }(|q \cdot(n-\bar{n})| / \nu)^{-\eta},
\end{aligned}
$$

where $\eta$ is the regularization parameter and $\nu_{+}, \nu_{-}$, and $\nu$ are regularization scales. The term $|q \cdot(n-\bar{n})|$ in the soft factor reduces to $|q \cdot \bar{n}|$ in the $n$ collinear region and to $|q . n|$ in the $\bar{n}$ collinear region, so the essential difference between the three factors in Eq. (14) is in the regularization scales. They are constrained by an equation that depends on the application. In most previous cases, the equation was either $\nu_{+} \nu_{-}=\nu^{2}$ or $\nu_{+} \nu_{-}=-\nu^{2}$. 


\section{B. Hard contribution}

The contribution to the LP form factor from the hard region in which $q^{\mu}$ is order $Q$ is

$$
\begin{aligned}
\mathcal{F}_{\text {hard }}^{\mathrm{LP}}(\hat{s})= & \frac{2 i g_{s}^{2} y_{b}}{D-2} \int_{q} \frac{1}{\left[(q+\tilde{P})^{2}+i \epsilon\right]\left[q^{2}+i \epsilon\right]\left[\left(q-p_{3}\right)^{2}+i \epsilon\right]} \\
& \times\left((5-D) q^{2}-4 \frac{\left(\tilde{P}+p_{3}\right) \cdot q p_{3} \cdot q}{\tilde{P} \cdot p_{3}}+2(D-3) p_{3} \cdot q+(D-2) \tilde{P} \cdot p_{3}\right) .
\end{aligned}
$$

The 4-momentum $P$ of the Higgs has been replaced by a light-like 4-vector $\tilde{P}$ whose 3 -vector component is collinear to $\boldsymbol{P}$ and whose normalization is given by $2 \tilde{P} \cdot p_{3}=\hat{s}$. The integral in Eq. (15) can be calculated analytically. A Laurent expansion in $\epsilon$ gives

$\mathcal{F}_{\text {hard }}^{\mathrm{LP}}(\hat{s})=-\frac{g_{s}^{2} y_{b}}{16 \pi^{2}}\left[\frac{-\hat{s}-i \epsilon}{\mu^{2}}\right]^{-\epsilon}\left(\frac{1}{\epsilon^{2}}+\frac{2}{\epsilon}+6-\frac{\pi^{2}}{6}\right)$.

The poles in $\epsilon$ have an infrared origin, but they can be transformed into ultraviolet poles in $\epsilon$ by adding integrals that have no scale and therefore vanish with dimensional regularization.

\section{Higgs collinear contribution}

As shown in Ref. [42], the scales $Q$ and $M$ in the Higgs collinear contribution to the LP form factor can be separated by expressing it as an integral over the relative longitudinal momentum fraction $\zeta$ of the collinear $b$ and $\bar{b}$ that form the Higgs:

$$
\mathcal{F}_{H \text { coll }}^{\mathrm{LP}}=\int_{-1}^{+1} d \zeta \tilde{\mathcal{F}}_{b \bar{b}_{1 V}+g}(\zeta) d_{b \bar{b}_{1 V} \rightarrow H}(\zeta) .
$$

The integrand is the product of a hard form factor $\tilde{\mathcal{F}}_{b \bar{b}_{1 V}+g}$ for producing a collinear $b \bar{b}$ pair in the color-singlet Lorentz-vector $(1 V)$ channel plus a gluon and a distribution amplitude $d_{b \bar{b}_{1 V} \rightarrow H}$ for a $b \bar{b}$ pair in the Higgs. The hard form factor depends only on the scale $Q$. The distribution amplitude depends on the scale $M$. With rapidity regularization, it also depends logarithmically on P.n.

The hard form factor in Eq. (17) is derived from the amplitude $\mathcal{T}^{\mu \nu}$ for $g^{*} \rightarrow b \bar{b}+g$, which is given by the sum of the two diagrams in Fig. 2. Since we only want the
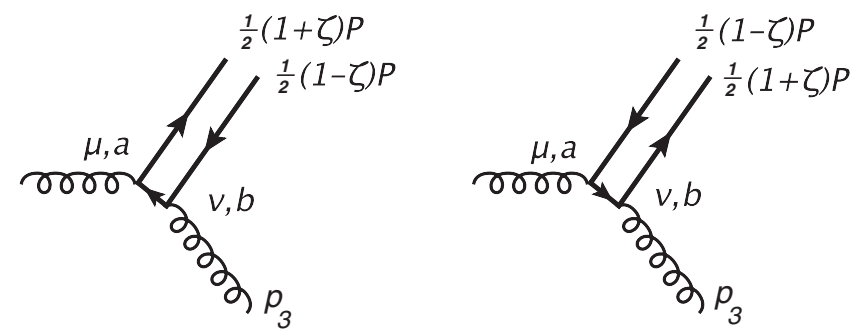

FIG. 2. Feynman diagrams for the tensor amplitude $\mathcal{T}^{\mu \nu}$ for $g^{*} \rightarrow b \bar{b}+g$ at LO. leading power, we can set $m_{b}=0$. We need the amplitude for producing $b$ and $\bar{b}$ in the color-singlet Lorentz-vector $(1 V)$ channel and with collinear momenta $\frac{1}{2}(1+\zeta) \tilde{P}$ and $\frac{1}{2}(1-\zeta) \tilde{P}$ plus a real gluon with momentum $p_{3}$. The $b \bar{b}$ pair can be projected onto the color-singlet state by tracing over the color indices of $b$ and $\bar{b}$ and dividing by $\sqrt{N_{c}}$, where $N_{c}=3$ is the number of quark colors. The $b \bar{b}$ pair can be projected onto the Lorentz-vector channel by replacing the outer product $v \bar{u}$ of the $\bar{b}$ and $b$ spinors by $\tilde{P}$. The $1 V$ contribution to the tensor amplitude is

$$
\begin{aligned}
\mathcal{T}_{1 V}^{\mu \nu}( & \left.P, p_{3}\right) \\
= & -\frac{4 g_{s}^{2}}{\sqrt{N_{c}}}\left(\frac{\tilde{P} \cdot p_{3} g^{\mu \nu}-\left(\tilde{P}^{\mu} p_{3}^{\nu}+p_{3}^{\mu} \tilde{P}^{\nu}\right)-(1-\zeta) \tilde{P}^{\mu} \tilde{P}^{\nu}}{(1-\zeta) \tilde{P} \cdot p_{3}}\right. \\
& -(\zeta \rightarrow-\zeta))
\end{aligned}
$$

The hard form factor for $g^{*} \rightarrow b \bar{b}_{1 V}+g$ is obtained by the contraction in Eq. (3) of the tensor $\mathcal{T}_{1 V}^{\mu \nu}$ in Eq. (18), with $P$ replaced by $\tilde{P}$. We choose to move a factor $1 /\left(1-\zeta^{2}\right)$ from the hard form factor to the distribution amplitude to allow the poles in the regularization parameters to be made explicit. A canceling factor $1-\zeta^{2}$ must appear in the hard form factor. We choose to also move the factor $1 /\left(\sqrt{N_{c}} m_{b}\right)$ to the distribution amplitude to simplify the expressions for both the hard form factor and the distribution amplitude. The resulting expression for the hard form factor is

$$
\tilde{\mathcal{F}}_{b \bar{b}_{1 V}+g}(\zeta)=-2 g_{s}^{2} \zeta
$$

The distribution amplitude for $b \bar{b}_{1 V} \rightarrow H$ in Eq. (17) is a function of the relative longitudinal momentum fraction $\zeta$ that describes how the longitudinal momentum of the Higgs is distributed between a $b$ and a $\bar{b}$. The distribution amplitude can be calculated by using ingredients from the Feynman rules for double-parton fragmentation functions in Ref. [53]. A fragmentation function can be expressed as the sum of cut diagrams that are products of an amplitude and the complex conjugate of an amplitude. The amplitude for $b \bar{b}$ fragmentation into a specific final state is the amplitude for that final state to be produced by sources that create the $b$ and the $\bar{b}$ in a specified color and Lorentz 
channel with relative longitudinal momentum fraction $\zeta$. The sources are the end points of eikonal lines that extend to future infinity. The Feynman rule for the sources that create the $b \bar{b}$ pair in the $1 V$ channel is the product of a color matrix, a Dirac matrix, and a delta function that are given in Ref. [42].

The leading-order diagram for the distribution amplitude for a $b \bar{b}$ pair in the Higgs is shown in Fig. 3. The expression for the distribution amplitude is $\sqrt{N_{c}} y_{b} m_{b} \zeta d(\zeta)$, where the function $d(\zeta)$ is

$$
d(\zeta)=-i \int_{q} \frac{\delta(\zeta-2 q . n / P . n)}{\left[\left(\frac{1}{2} P+q\right)^{2}-m_{b}^{2}+i \epsilon\right]\left[\left(\frac{1}{2} P-q\right)^{2}-m_{b}^{2}+i \epsilon\right]} .
$$

We have suppressed the rapidity regularization factor and zero-bin subtractions for the integral over the loop momentum $q$. The rapidity regularization factor is the product of two factors like that in Eq. (14b) with $q$ replaced by $\frac{1}{2} P+q$ and by $\frac{1}{2} P-q$. Multiplying by the factors $1 /\left(\sqrt{N_{c}} m_{b}\right)$ and $1 /\left(1-\zeta^{2}\right)$ that were removed from the form factor for $g^{*} \rightarrow b \bar{b}_{1 V}+g$ in Eq. (19), we obtain the distribution amplitude

$$
d_{b \bar{b}_{1 V} \rightarrow H}(\zeta)=y_{b} \zeta \frac{d(\zeta)}{1-\zeta^{2}}
$$

The function $d(\zeta)$ is calculated with rapidity regularization and with appropriate zero-bin subtractions in the appendix of Ref. [42]. It has ultraviolet divergences that can be made explicit as poles in the regularization parameters $\epsilon$ and $\eta$ if the function is divided by $1-\zeta^{2}$ :

$$
\begin{aligned}
\frac{d(\zeta)}{1-\zeta^{2}}= & \frac{1}{32 \pi^{2}}\left[\frac{\mu^{2}}{m_{b}^{2}}\right]^{\epsilon}\left[\frac{P . n}{\nu_{1}}\right]^{-2 \eta} \frac{1}{\epsilon} \\
& \times\left(-\frac{1}{2 \eta} \delta\left(1-\zeta^{2}\right)+\frac{1}{\left(1-\zeta^{2}\right)_{+}}\right) \\
& \times\left[1-\left(1-\zeta^{2}\right) r^{2}-i \epsilon\right]^{-\epsilon} .
\end{aligned}
$$

We have set the rapidity regularization scale to $\nu_{1}$. The plus distribution can be defined by giving the integral of the

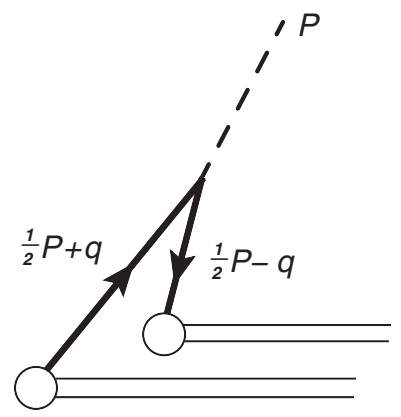

FIG. 3. Feynman diagram for the distribution amplitude for $b \bar{b}_{1 V} \rightarrow H$ at LO. product of the distribution and a smooth function $f(z)$ over the closed interval $-1 \leq z \leq+1$ :

$$
\begin{aligned}
& \int_{-1}^{+1} d \zeta g(\zeta)_{+} f(\zeta) \\
& \quad \equiv \int_{-1}^{+1} d \zeta g(\zeta) \frac{f(\zeta)+f(-\zeta)-f(1)-f(-1)}{2}
\end{aligned}
$$

The distribution amplitude in Eq. (21) is

$$
\begin{aligned}
d_{b \bar{b}_{1 V} \rightarrow H}(\zeta)= & \frac{y_{b}}{32 \pi^{2}}\left[\frac{\mu^{2}}{m_{b}^{2}}\right]^{\epsilon}\left[\frac{P . n}{\nu_{1}}\right]^{-2 \eta} \\
& \times \zeta\left\{\frac{1}{\epsilon}\left(-\frac{1}{2 \eta} \delta\left(1-\zeta^{2}\right)+\frac{1}{\left(1-\zeta^{2}\right)_{+}}\right)\right. \\
& \left.-\frac{\log \left(1-\left(1-\zeta^{2}\right) r^{2}-i \epsilon\right)}{1-\zeta^{2}}\right\} .
\end{aligned}
$$

The Higgs collinear contribution to the LP form factor is obtained by evaluating the integral over $\zeta$ in Eq. (17):

$$
\begin{aligned}
\mathcal{F}_{H \text { coll }}^{\mathrm{LP}}\left(m_{b}^{2}, \tilde{P} . n\right)= & \frac{g_{s}^{2} y_{b}}{16 \pi^{2}}\left[\frac{\mu^{2}}{m_{b}^{2}}\right]^{\epsilon}\left\{\frac{1}{\epsilon}\left(\frac{1}{2 \eta}-\log \frac{\tilde{P} . n}{\nu_{1}}+2\right)\right. \\
& \left.+\int_{-1}^{+1} d \zeta \zeta^{2} \frac{\log \left(1-\left(1-\zeta^{2}\right) r^{2}-i \epsilon\right)}{1-\zeta^{2}}\right\} .
\end{aligned}
$$

It depends logarithmically on $\tilde{P} . n$. The remaining integral over $\zeta$ is

$$
\begin{array}{rl}
\int_{-1}^{+1} & d \zeta \zeta^{2} \frac{\log \left(1-\left(1-\zeta^{2}\right) r^{2}-i \epsilon\right)}{1-\zeta^{2}} \\
= & \frac{1}{2}\left(\log \frac{r+\sqrt{r^{2}-1}}{r-\sqrt{r^{2}-1}}-i \pi\right)^{2} \\
& -\frac{2 \sqrt{r^{2}-1}}{r}\left(\log \frac{r+\sqrt{r^{2}-1}}{r-\sqrt{r^{2}-1}}-i \pi\right)+4
\end{array}
$$

\section{Gluon collinear contribution}

As shown in Ref. [42], the scales $Q$ and $M$ in the gluon collinear contribution to the LP form factor can be separated by expressing it as an integral over the relative longitudinal momentum fraction $\zeta$ :

$$
\mathcal{F}_{g \mathrm{coll}}^{\mathrm{LP}}=\int_{-1}^{+1} d \zeta \tilde{\mathcal{F}}_{H+b \bar{b}_{8 T}}(\zeta) d_{b \bar{b}_{8 T} \rightarrow g}(\zeta) .
$$

The integrand is the product of the hard form factor $\tilde{\mathcal{F}}_{H+b \bar{b}_{8 T}}$ for producing a Higgs plus a collinear $b \bar{b}$ pair in the color-octet Lorentz-tensor (8T) channel and the distribution amplitude $d_{b \bar{b}_{8 T} \rightarrow g}$ for a $b \bar{b}$ pair in a real gluon. The hard form factor depends only on the scale $Q$. The distribution amplitude depends on the scale $M$. With 
rapidity regularization, it also depends logarithmically on $p_{3} \cdot \bar{n}$.

The hard form factor in Eq. (27) is determined from the amplitude $\mathcal{T}^{\mu}$ for $g^{*} \rightarrow H+b \bar{b}$, which is given by the sum of the two diagrams in Fig. 4. Since we only want the leading power, we can set $m_{b}=0$. We need the amplitude for producing $b$ and $\bar{b}$ in the color-octet Lorentz-tensor (8T) channel with collinear momenta $\frac{1}{2}(1+\zeta) p_{3}$ and $\frac{1}{2}(1-\zeta) p_{3}$ plus a Higgs with momentum $\tilde{P}$. The $b \bar{b}$ pair can be projected onto a color-octet state with the color index $a$ by tracing the amplitude with the color matrix $\sqrt{2} T^{a}$. The $b \bar{b}$ pair can be projected onto the Lorentz-tensor channel with a Lorentz index $\nu$ by replacing the outer spinor product $v \bar{u}$ by $\not p_{3} \gamma_{\perp}^{\nu}$, where $\gamma_{\perp}^{\nu}=g_{\perp}^{\nu \alpha} \gamma_{\alpha}$ are Dirac matrices that are perpendicular to $n$ and $\bar{n}$, and $g_{\perp}^{\nu \alpha}$ is the perpendicular metric tensor:

$$
g_{\perp \alpha \beta}=g_{\alpha \beta}-\frac{n_{\alpha} \bar{n}_{\beta}+\bar{n}_{\alpha} n_{\beta}}{n \cdot \bar{n}} .
$$

The $8 T$ contribution to the vector amplitude $\mathcal{T}^{\mu}$ defines the tensor amplitude

$$
\mathcal{T}_{8 T}^{\mu \nu}\left(P, p_{3}\right)=-4 \sqrt{2} g_{s} y_{b} g_{\perp}^{\mu \nu}\left(\frac{1}{1+\zeta}+\frac{1}{1-\zeta}\right) .
$$

The hard form factor for $g^{*} \rightarrow H+b \bar{b}_{8 T}$ can be obtained by the contraction in Eq. (3) of the tensor $\mathcal{T}_{8 T}^{\mu \nu}$ in Eq. (29), with $P$ replaced by $\tilde{P}$. We choose to move a factor $1 /\left(1-\zeta^{2}\right)$ to the distribution amplitude to allow the poles in the regularization parameters to be made explicit. A canceling factor $1-\zeta^{2}$ must appear in the hard form factor. We also choose to move the factor $\sqrt{2} / m_{b}$ to the distribution amplitude to simplify the expressions for both the hard form factor and the distribution amplitude. The resulting expression for the hard form factor is

$$
\tilde{\mathcal{F}}_{H+b \bar{b}_{8 T}}(\zeta)=-2 g_{s} y_{b} .
$$

The distribution amplitude for $b \bar{b}_{8 T} \rightarrow g$ in Eq. (27) is a function of the relative longitudinal momentum fraction $\zeta$ that describes how the longitudinal momentum of the real
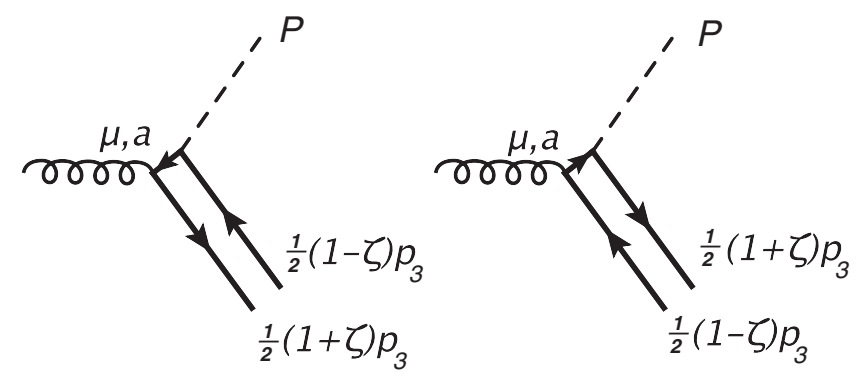

FIG. 4. Feynman diagrams for the tensor amplitude $\mathcal{T}^{\mu a, b}$ for $g^{*} \rightarrow H+b \bar{b}$ at LO. gluon is distributed between a $b$ and a $\bar{b}$. It can be calculated from the diagram in Fig. 5 by using ingredients from the Feynman rules for double-parton fragmentation functions in Ref. [53]. The Feynman rule for the sources that create the $b \bar{b}$ pair in the $8 T$ channel is the product of a color matrix, a Dirac matrix, and a delta function that are given in Ref. [42].

The leading-order diagram for the distribution amplitude for a $b \bar{b}$ pair in a real gluon is shown in Fig. 5. The amplitude for the source to produce a real gluon with transverse polarization vector in the same direction as the source and with the same color index as the source is $\left(g_{s} m_{b} / \sqrt{2}\right) d_{0}(\zeta)$, where the function $d_{0}(\zeta)$ is

$d_{0}(\zeta)=-i \int_{q} \frac{\delta\left(\zeta-2 q \cdot \bar{n} / p_{3} \cdot \bar{n}\right)}{\left[\left(\frac{1}{2} p_{3}+q\right)^{2}-m_{b}^{2}+i \epsilon\right]\left[\left(\frac{1}{2} p_{3}-q\right)^{2}-m_{b}^{2}+i \epsilon\right]}$.

We have suppressed rapidity regularization factors and zero-bin subtractions in the integral over the loop momentum $q$. Multiplying by the factors $\sqrt{2} / m_{b}$ and $1 /\left(1-\zeta^{2}\right)$ that were removed from the form factor for $g^{*} \rightarrow H+b \bar{b}_{8 T}$, we obtain the distribution amplitude

$$
d_{b \bar{b}_{8 T} \rightarrow g}(\zeta)=g_{s} \frac{d_{0}(\zeta)}{1-\zeta^{2}} .
$$

The function $d_{0}(\zeta) /\left(1-\zeta^{2}\right)$ with rapidity regularization can be obtained from the function $d(\zeta) /\left(1-\zeta^{2}\right)$ in Eq. (22) by setting $r=0$ and replacing P.n with $p_{3} . \bar{n}$. The distribution amplitude in Eq. (32) is

$$
\begin{aligned}
d_{b \bar{b}_{8 T} \rightarrow g}(\zeta)= & \frac{g_{s}}{32 \pi^{2}}\left[\frac{\mu^{2}}{m_{b}^{2}}\right]^{\epsilon}\left[\frac{p_{3} \cdot \bar{n}}{\nu_{3}}\right]^{-2 \eta} \frac{1}{\epsilon} \\
& \times\left\{-\frac{1}{2 \eta} \delta\left(1-\zeta^{2}\right)+\frac{1}{\left(1-\zeta^{2}\right)_{+}}\right\} .
\end{aligned}
$$

We have set the rapidity regularization scale to $\nu_{3}$. The poles in $\epsilon$ and $\eta$ have ultraviolet origins. The plus distribution is defined in Eq. (23).

The gluon collinear contribution to the LP form factor is obtained by evaluating the integral over $\zeta$ in Eq. (27):

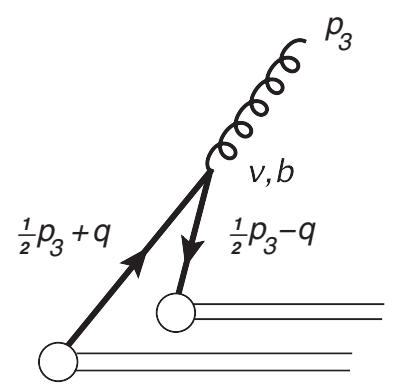

FIG. 5. Feynman diagram for the distribution amplitude for $b \bar{b}_{8 T} \rightarrow g$ at LO. 
$\mathcal{F}_{g \text { coll }}^{\mathrm{LP}}\left(m_{b}^{2}, p_{3} \cdot \bar{n}\right)=\frac{g_{s}^{2} y_{b}}{16 \pi^{2}}\left[\frac{\mu^{2}}{m_{b}^{2}}\right]^{\epsilon} \frac{1}{\epsilon}\left(\frac{1}{2 \eta}-\log \frac{p_{3} \cdot \bar{n}}{\nu_{3}}\right)$.

It depends logarithmically on $p_{3} \cdot \bar{n}$.

\section{E. Soft contribution}

In the soft region of the loop momentum $q$, all the components of $q$ are order $M$. The soft contribution to the LP form factor with rapidity regularization is

$$
\begin{aligned}
\mathcal{F}_{\text {soft }}^{\mathrm{LP}}= & 2 i g_{s}^{2} y_{b} \int_{q} \frac{\tilde{P} \cdot p_{3}}{[2 \tilde{P} \cdot q+i \epsilon]\left[q^{2}-m_{b}^{2}+i \epsilon\right]\left[-2 p_{3} \cdot q+i \epsilon\right]} \\
& \times\left[\frac{|q \cdot(n-\bar{n})|}{\nu}\right]^{-2 \eta} .
\end{aligned}
$$

The 4-momentum $P$ of the Higgs has been replaced by the light-like 4 -vector $\tilde{P}$. The rapidity divergences from the two denominators $2 \tilde{P} . q$ and $-2 p_{3} . q$ have been regularized by multiplying the integrand by two identical copies of the factor in Eq. (14c). The integral over $q$ in Eq. (35) gives ultraviolet poles in $\eta$ and in $\epsilon+\eta$. After a Laurent expansion in $\eta$, the soft contribution reduces to

$\mathcal{F}_{\text {soft }}^{\mathrm{LP}}\left(m_{b}^{2}\right)=-\frac{g_{s}^{2} y_{b}}{16 \pi^{2}}\left[\frac{\mu^{2}}{m_{b}^{2}}\right]^{\epsilon}\left(\frac{1}{\epsilon \eta}-\frac{1}{\epsilon^{2}}+\frac{1}{\epsilon} \log \frac{\nu^{2}}{m_{b}^{2}}+\frac{\pi^{2}}{6}\right)$.

\section{F. LP form factor}

In the sum of the Higgs collinear contribution in Eq. (25), the gluon collinear contribution in Eq. (34), and the soft contribution in Eq. (36), the ultraviolet poles in $\eta$ from rapidity divergences cancel. The only divergences that remain are double and single poles in $\epsilon$ :

$$
\begin{aligned}
\mathcal{F}_{H \text { coll }}^{\mathrm{LP}} & +\mathcal{F}_{\text {gcoll }}^{\mathrm{LP}}+\mathcal{F}_{\text {soft }}^{\mathrm{LP}} \\
= & \frac{g_{s}^{2} y_{b}}{16 \pi^{2}}\left[\frac{\mu^{2}}{m_{b}^{2}}\right]^{\epsilon}\left\{\frac{1}{\epsilon^{2}}-\frac{1}{\epsilon}\left(\log \frac{\tilde{P} \cdot n}{\nu_{1}}+\log \frac{p_{3} \cdot \bar{n}}{\nu_{3}}+\log \frac{\nu^{2}}{m_{b}^{2}}-2\right)\right. \\
& \left.-\frac{\pi^{2}}{6}+\int_{-1}^{+1} d \zeta \zeta^{2} \frac{\log \left(1-\left(1-\zeta^{2}\right) r^{2}-i \epsilon\right)}{1-\zeta^{2}}\right\} .
\end{aligned}
$$

Upon adding the hard contribution in Eq. (16) to get the complete LP form factor, the double poles in $\epsilon$ cancel:

$$
\begin{aligned}
\mathcal{F}^{\mathrm{LP}}= & \frac{g_{s}^{2} y_{b}}{16 \pi^{2}}\left[\frac{\mu^{2}}{m_{b}^{2}}\right]^{\epsilon}\left\{-\frac{1}{\epsilon}\left(\log \frac{\tilde{P} \cdot n p_{3} \cdot \bar{n}}{-\hat{s}-i \epsilon}+\log \frac{\nu^{2}}{\nu_{1} \nu_{3}}\right)\right. \\
& -\frac{1}{2} \log ^{2} \frac{-\hat{s}-i \epsilon}{m_{b}^{2}}+2 \log \frac{-\hat{s}-i \epsilon}{m_{b}^{2}} \\
& \left.-6+\int_{-1}^{+1} d \zeta \zeta^{2} \frac{\log \left(1-\left(1-\zeta^{2}\right) r^{2}-i \epsilon\right)}{1-\zeta^{2}}\right\} .
\end{aligned}
$$

Since $\tilde{P} . n p_{3} \cdot \bar{n}=\hat{s}$, the single poles in $\epsilon$ cancel provided the rapidity regularization scales satisfy

$$
\nu_{1} \nu_{3}=e^{+i \pi} \nu^{2} .
$$

This nontrivial constraint on the rapidity regularization scales is necessary to separate the contributions from the different regions. In Ref. [42], this constraint was verified by comparing with the result using analytic regularization. It would be preferable to derive it from deeper theoretical considerations. The final result for the LP form factor is obtained by inserting the integral in Eq. (26) into Eq. (38). It agrees with the result in Eq. (6) obtained from Refs. [5,6].

\section{RENORMALIZED FACTORIZATION FORMULA}

In this section, we present a renormalized factorization formula for the LP form factor in which each of the pieces is separately finite. Such a form would be necessary for the resummation of large logarithms since solving evolution equations numerically requires finite inputs as initial conditions. The ultraviolet divergences in each of the regularized pieces of the factorization formula are removed by the minimal subtraction of the poles from dimensional regularization and from rapidity regularization. This renormalization procedure is equivalent to canceling the divergences by moving the divergent terms between different regions. The renormalized contribution from each region depends on the renormalization scheme, but the sum over all regions is scheme independent. We also define an $\mathrm{LP} b$ form factor in which the errors are reduced from order $m_{H}^{2} / Q^{2}$ to order $m_{b}^{2} / Q^{2}$.

\section{A. LP form factor}

The factorization formula for the LP form factor is given in a schematic form in Eq. (11). The explicit form of the renormalized factorization formula is

$$
\begin{aligned}
& \mathcal{F}^{\mathrm{LP}}\left(\hat{s}, m_{b}^{2}, m_{H}^{2}\right) \\
& \equiv \tilde{\mathcal{F}}_{H+g}(\hat{s})+\int_{-1}^{+1} d \zeta \tilde{\mathcal{F}}_{b \bar{b}_{1 V}+g}(\zeta) d_{b \bar{b}_{1 V} \rightarrow H}\left(\zeta ; m_{b}^{2}, m_{H}^{2}, P . n\right) \\
& \quad+\int_{-1}^{+1} d \zeta \tilde{\mathcal{F}}_{H+b \bar{b}_{8 T}}(\zeta) d_{b \bar{b}_{8 T} \rightarrow g}\left(\zeta ; m_{b}^{2}, p_{3} . \bar{n}\right)+\mathcal{F}_{\text {endpt }}\left(m_{b}^{2}\right) .
\end{aligned}
$$

All the dependences on physical scales are indicated explicitly by the arguments in Eq. (40). Each of the individual pieces in the factorization formula is given below.

The regularized hard contribution to the LP form factor is given in Eq. (16). We define the renormalized contribution from the direct production of $H+g$ by minimal subtraction of the poles in $\epsilon$ :

$$
\tilde{\mathcal{F}}_{H+g}(\hat{s})=\frac{g_{s}^{2} y_{b}}{16 \pi^{2}}\left(-\frac{1}{2} \log ^{2} \frac{-\hat{s}-i \epsilon}{\mu^{2}}+2 \log \frac{-\hat{s}-i \epsilon}{\mu^{2}}+\frac{\pi^{2}}{6}-6\right) .
$$


With the measure of the dimensionally regularized momentum integral defined in Eq. (10), the minimal subtraction of the poles in $\epsilon$ corresponds to the modified minimal subtraction $(\overline{\mathrm{MS}})$ renormalization scheme. The renormalized hard contribution depends logarithmically on $\hat{s}$.

The Higgs collinear contribution to the LP form factor is given by the integral over the relative longitudinal momentum fraction $\zeta$ in Eq. (17). The hard form factor for $g^{*} \rightarrow$ $b \bar{b}_{1 V}+g$ is given in Eq. (19). The distribution amplitude for $b \bar{b}_{1 V} \rightarrow H$ with rapidity regularization is given in Eq. (24). We define a renormalized distribution amplitude by minimal subtraction of the ultraviolet poles in $\eta$ and in $\epsilon$ :

$$
\begin{aligned}
d_{b \bar{b}_{1 V} \rightarrow H}(\zeta)= & \frac{y_{b}}{32 \pi^{2}} \zeta\left[\log \frac{\mu^{2}}{m_{b}^{2}}\left(\log \frac{P . n}{\nu_{1}} \delta\left(1-\zeta^{2}\right)+\frac{1}{\left(1-\zeta^{2}\right)_{+}}\right)\right. \\
& \left.-\frac{\log \left(1-\left(1-\zeta^{2}\right) r^{2}-i \epsilon\right)}{1-\zeta^{2}}\right],
\end{aligned}
$$

where $r=m_{H} /\left(2 m_{b}\right)$. The distribution amplitude depends logarithmically on P.n. The first integral over $\zeta$ in Eq. (40) can be evaluated by inserting the renormalized distribution amplitude in Eq. (42). The result agrees with that obtained by the minimal subtraction of the poles in the regularized integral in Eq. (25).

The gluon collinear contribution to the LP form factor is given by the integral over $\zeta$ in Eq. (27). The form factor for $g^{*} \rightarrow H+b \bar{b}_{8 T}$ is given in Eq. (30). The distribution amplitude for $b \bar{b}_{8 T} \rightarrow g$ with rapidity regularization is given in Eq. (33). We define a renormalized distribution amplitude by minimal subtraction of the ultraviolet poles in $\eta$ and in $\epsilon$ :

$$
d_{b \bar{b}_{8 T} \rightarrow g}(\zeta)=\frac{g_{s}}{32 \pi^{2}} \log \frac{\mu^{2}}{m_{b}^{2}}\left(\log \frac{p_{3} \cdot \bar{n}}{\nu_{3}} \delta\left(1-\zeta^{2}\right)+\frac{1}{\left(1-\zeta^{2}\right)_{+}}\right) .
$$

The distribution amplitude depends logarithmically on $p_{3} . \bar{n}$. The second integral over $\zeta$ in Eq. (40) can be evaluated by inserting the renormalized distribution amplitude in Eq. (43). The result agrees with that obtained by the minimal subtraction of the poles in the regularized integral in Eq. (34).

The soft contribution to the LP form factor using rapidity regularization is given in Eq. (36). We define the renormalized end point contribution by minimal subtraction of the ultraviolet poles in $\eta$ and in $\epsilon$ :

$\mathcal{F}_{\text {endpt }}\left(m_{b}^{2}\right)=\frac{g_{s}^{2} y_{b}}{16 \pi^{2}}\left(\frac{1}{2} \log ^{2} \frac{\mu^{2}}{m_{b}^{2}}-\log \frac{\mu^{2}}{m_{b}^{2}} \log \frac{\nu^{2}}{m_{b}^{2}}-\frac{\pi^{2}}{6}\right)$.

The end point contribution depends logarithmically on $m_{b}$.

The sum of the four terms in Eq. (40) reproduces the LP form factor in Eq. (6). The logarithms of P.n from the
Higgs collinear term and $p_{3} \cdot \bar{n}$ from the gluon collinear term combine to give a logarithm of $\hat{s}$. The last three terms in Eq. (40) depend on the rapidity regularization scales $\nu_{1}, \nu_{3}$, and $\nu$. The dependence on these scales cancels upon using the relation among $\nu_{1}, \nu_{3}$, and $\nu$ in Eq. (39). All four terms in Eq. (40) depend on the dimensional regularization scale $\mu$. The dependence on $\mu$ cancels when the four terms are added.

\section{B. Improved mass dependence}

The leading errors in the LP form factor come from the omission of terms in the form factor that are suppressed either by $m_{b}^{2} / Q^{2}$ or by $m_{H}^{2} / Q^{2}$. Since $m_{H}$ is an order of magnitude larger than $2 m_{b}$, one should be able to improve the accuracy by keeping the leading terms of the expansion in $m_{b}^{2} / Q^{2}$ without expanding in $m_{H}^{2} / Q^{2}$. This will not change the parametric dependence of the error, which still decreases as $1 / Q^{2}$, but one might hope for a decrease in the numerical size of the error by two orders of magnitude. We refer to the leading term in the expansion of the form factor in powers of $m_{b}^{2} / Q^{2}$ as the $\mathrm{LP} b$ form factor, and we denote it by $\mathcal{F}^{\mathrm{LP} b}\left(\hat{s}, m_{b}^{2}, m_{H}^{2}\right)$. It can be defined as the leading power in $M^{2} / Q^{2}$, where the hard scale is $Q \sim P_{T}, \sqrt{\hat{s}}$, and the soft scale is $M \sim m_{b}$, but $m_{H}$ is an arbitrary intermediate scale. The $\mathrm{LP} b$ form factor includes terms of all powers in $m_{H}^{2} / Q^{2}$ that are not suppressed by $m_{b}^{2} / Q^{2}$ and therefore has an error of order $m_{b}^{2} / Q^{2}$. We will show that it can be expressed in the same form as the factorization formula for the LP form factor in Eq. (40), with the only change being that the hard form factor $\tilde{\mathcal{F}}_{H+g}(\hat{s})$ is replaced by an $m_{H}$-dependent hard form factor $\tilde{\mathcal{F}}_{H+g}^{(H)}\left(\hat{s}, m_{H}^{2}\right)$. The schematic form of the $\mathrm{LP} b$ factorization formula is

$$
\begin{aligned}
\mathcal{F}^{\mathrm{LP} b} & {[H+g] } \\
\equiv & \tilde{\mathcal{F}}(H)[H+g]+\tilde{\mathcal{F}}\left[b \bar{b}_{1 V}+g\right] \otimes d\left[b \bar{b}_{1 V} \rightarrow H\right] \\
& +\tilde{\mathcal{F}}\left[H+b \bar{b}_{8 T}\right] \otimes d\left[b \bar{b}_{8 T} \rightarrow g\right]+\mathcal{F}_{\text {endpt }}[H+g] .
\end{aligned}
$$

We first explain how the $m_{H}$-dependent hard form factor $\tilde{\mathcal{F}}^{(H)}[H+g]$ in Eq. (45) can be obtained. The schematic factorization formula for the LP form factor $\mathcal{F}^{\mathrm{LP}}[H+g]$ in Eq. (11) can be solved for the hard form factor $\tilde{\mathcal{F}}[H+g]$. Since this hard form factor does not depend on $m_{H}$ or $m_{b}$, it can be expressed as a double limit as $m_{b} \rightarrow 0$ and $m_{H} \rightarrow 0$ :

$$
\begin{aligned}
\tilde{\mathcal{F}}[H+g]= & {\left[\mathcal{F}^{\mathrm{LP}}[H+g]-\tilde{\mathcal{F}}\left[b \bar{b}_{1 V}+g\right] \otimes d\left[b \bar{b}_{1 V} \rightarrow H\right]\right.} \\
& -\tilde{\mathcal{F}}\left[H+b \bar{b}_{8 T}\right] \otimes d\left[b \bar{b}_{8 T} \rightarrow g\right] \\
& \left.-\mathcal{F}_{\text {endpt }}[H+g]\right]_{\substack{m_{b} \rightarrow 0 \\
m_{H} \rightarrow 0}}
\end{aligned}
$$

The LP form factor differs from the full form factor only by terms with higher powers of $m_{H}$ and $m_{b}$, so $\mathcal{F}^{\mathrm{LP}}[H+g]$ 
can be replaced by $\mathcal{F}[H+g]$ inside the limits. We define a regularized $m_{H}$-dependent hard form factor by making this replacement and then removing the limit $m_{H} \rightarrow 0$ :

$$
\begin{aligned}
\tilde{\mathcal{F}}^{(H)}[H+g] \equiv & {\left[\mathcal{F}[H+g]-\tilde{\mathcal{F}}\left[b \bar{b}_{1 V}+g\right] \otimes d\left[b \bar{b}_{1 V} \rightarrow H\right]\right.} \\
& -\tilde{\mathcal{F}}\left[H+b \bar{b}_{8 T}\right] \otimes d\left[b \bar{b}_{8 T} \rightarrow g\right] \\
& \left.-\mathcal{F}_{\text {endpt }}[H+g]\right]_{m_{b} \rightarrow 0} .
\end{aligned}
$$

All four terms on the right side have additional infrared divergences in the limit $m_{b} \rightarrow 0$. The additional infrared divergences cancel in the sum of the four terms, leaving the same infrared poles in $\epsilon$ as in the regularized hard form factor in Eq. (16).

We now proceed to show that the errors in the $\mathrm{LP} b$ form factor defined by Eq. (45) are order $m_{b}^{2} / Q^{2}$. The difference between the $\mathrm{LP} b$ form factor and the LP form factor in Eq. (11) is equal to the difference $\tilde{\mathcal{F}}^{(H)}[H+g]-\tilde{\mathcal{F}}[H+g]$ between the hard form factors, which is order $m_{H}^{2} / \hat{s}$. Since the error in the LP form factor decreases as $1 / \hat{s}$, the error in the $\mathrm{LP} b$ form factor must also decrease as $1 / \hat{s}$. By inserting the expression for $\tilde{\mathcal{F}}^{(H)}[H+g]$ in Eq. (47) into the expression for $\mathcal{F}^{\mathrm{LP} b}[H+g]$ in Eq. (45), we find that the difference between the $\mathrm{LP} b$ form factor and the full form factor can be expressed as

$$
\begin{aligned}
\mathcal{F}^{\mathrm{LP}} b & {[H+g]-\mathcal{F}[H+g] } \\
= & \left(\left.\mathcal{F}[H+g]\right|_{m_{b}=0}-\mathcal{F}[H+g]\right) \\
& +\tilde{\mathcal{F}}\left[b \bar{b}_{1 V}+g\right] \otimes\left(d\left[b \bar{b}_{1 V} \rightarrow H\right]-\left.d\left[b \bar{b}_{1 V} \rightarrow H\right]\right|_{m_{b}=0}\right) \\
& +\tilde{\mathcal{F}}\left[H+b \bar{b}_{8 T}\right] \otimes\left(d\left[b \bar{b}_{8 T} \rightarrow g\right]-\left.d\left[b \bar{b}_{8 T} \rightarrow g\right]\right|_{m_{b}=0}\right) \\
& +\left(\mathcal{F}_{\text {endpt }}[H+g]-\left.\mathcal{F}_{\text {endpt }}[H+g]\right|_{m_{b}=0}\right) .
\end{aligned}
$$

Each term on the right side is 0 for $m_{b}=0$, so the right side is proportional to $m_{b}^{2}$. Since the error in the $\mathrm{LP} b$ form factor decreases as $1 / \hat{s}$, it must be order $m_{b}^{2} / \hat{s}$.

The additional infrared divergences in the terms on the right side of Eq. (47) could be regularized by the $b$ quark mass. This requires calculating all four terms on the right side of Eq. (47) with nonzero $m_{b}$ and then taking the limit $m_{b} \rightarrow 0$ at the end. The additional infrared divergences appear as logarithms of $m_{b}$, and they cancel between the four terms on the right side of Eq. (47). Calculating the full form factor $\mathcal{F}$ and then taking the limit $m_{b} \rightarrow 0$ still requires a calculation involving all three scales $\hat{s}, m_{H}$, and $m_{b}$. If this were necessary, the $\mathrm{LP} b$ form factor would have no calculational advantage over the full form factor. However instead of taking the limit $m_{b} \rightarrow 0$, the $m_{H}$-dependent hard form factor can be calculated more easily by setting $m_{b}=0$ from the beginning in all four terms. The additional infrared divergences from setting $m_{b}=0$ can be regularized using dimensional regularization. The additional poles in $\epsilon$ cancel in the sum of the four terms.

We proceed to calculate each of the four terms on the right side of Eq. (47) with $m_{b}=0$. The first term on the right side of Eq. (47) can be obtained by setting $m_{b}=0$ in the dimensionally regularized expression for the full form factor in Eq. (9):

$$
\begin{aligned}
\mathcal{F}\left(\hat{s}, 0, m_{H}^{2}\right) & \\
= & \frac{2 i g_{s}^{2} y_{b}}{D-2} \int_{q} \frac{1}{\left[(q+P)^{2}+i \epsilon\right]\left[q^{2}+i \epsilon\right]\left[\left(q-p_{3}\right)^{2}+i \epsilon\right]} \\
& \times\left((5-D) q^{2}-4 \frac{\left(P+p_{3}\right) \cdot q p_{3} \cdot q}{P \cdot p_{3}}+2(D-3) p_{3} \cdot q\right. \\
& \left.+(D-2) P \cdot p_{3}\right) .
\end{aligned}
$$

After evaluating the loop integral, we get

$$
\mathcal{F}\left(\hat{s}, 0, m_{H}^{2}\right)=\frac{g_{s}^{2} y_{b}}{16 \pi^{2}}\left[\frac{\mu^{2}}{m_{H}^{2}}\right]^{\epsilon} e^{i \pi \epsilon}\left[-\frac{1}{2} \log ^{2} \frac{\hat{s}+i \epsilon}{m_{H}^{2}}+\left(\frac{1}{\epsilon}+\frac{2 \hat{s}}{\hat{s}-m_{H}^{2}}\right) \log \frac{\hat{s}+i \epsilon}{m_{H}^{2}}-2\right] .
$$

The pole in $\epsilon$ has an infrared origin.

In the second term on the right side of Eq. (47), all the dependence on $m_{b}$ is in the function $d(\zeta)$ in the expression for the distribution amplitude $d_{b \bar{b}_{1 V} \rightarrow H}(\zeta)$ in Eq. (21). The expression for $d(\zeta)$ is obtained by setting $m_{b}=0$ in Eq. (20):

$$
d(\zeta)=-i \int_{q} \frac{\delta(\zeta-2 q \cdot n / P . n)}{\left[\left(\frac{1}{2} P+q\right)^{2}+i \epsilon\right]\left[\left(\frac{1}{2} P-q\right)^{2}+i \epsilon\right]} .
$$

The integral has no rapidity divergences, so dimensional regularization is sufficient. The analytic expression for the integral is

$$
d(\zeta)=\frac{1}{32 \pi^{2} \epsilon}\left[\frac{\mu^{2}}{m_{H}^{2}}\right]^{\epsilon}\left(e^{-i \pi} \frac{1-\zeta^{2}}{4}\right)^{-\epsilon}
$$

The poles in $\epsilon$ can be made explicit by using the expansion

$$
\frac{1}{1-\zeta^{2}}\left(\frac{1-\zeta^{2}}{4}\right)^{-\epsilon}=-\frac{\Gamma^{2}(1-\epsilon)}{\epsilon \Gamma(1-2 \epsilon)} \delta\left(1-\zeta^{2}\right)+\frac{1}{\left(1-\zeta^{2}\right)_{+}}-\epsilon\left(\frac{\log \left(1-\zeta^{2}\right)-2 \log 2}{1-\zeta^{2}}\right)_{+}+O\left(\epsilon^{2}\right) .
$$


The resulting expression for the regularized distribution amplitude in Eq. (21) is

$$
\left.d_{b \bar{b}_{1 V} \rightarrow H}(\zeta)\right|_{m_{b}=0}=\frac{y_{b} \zeta}{32 \pi^{2}}\left[\frac{\mu^{2}}{m_{H}^{2}}\right]^{\epsilon} e^{i \pi \epsilon}\left\{\left(-\frac{1}{\epsilon^{2}}+\frac{\pi^{2}}{6}\right) \delta\left(1-\zeta^{2}\right)+\frac{1}{\epsilon} \frac{1}{\left(1-\zeta^{2}\right)_{+}}-\left(\frac{\log \left(1-\zeta^{2}\right)-2 \log 2}{1-\zeta^{2}}\right)_{+}\right\} .
$$

Multiplying by the hard form factor in Eq. (19) and integrating over $\zeta$, we obtain

$$
\left.\int_{-1}^{+1} d \zeta \tilde{\mathcal{F}}_{b \bar{b}_{1 V}+g}(\zeta) d_{b \bar{b}_{1 V} \rightarrow H}(\zeta)\right|_{m_{b}=0}=\frac{g_{s}^{2} y_{b}}{16 \pi^{2}}\left[\frac{\mu^{2}}{m_{H}^{2}}\right]^{\epsilon} e^{i \pi \epsilon}\left(\frac{1}{\epsilon^{2}}+\frac{2}{\epsilon}-\frac{\pi^{2}}{6}+4\right) .
$$

In the third term on the right side of Eq. (47), all the dependence on $m_{b}$ is in the function $d_{0}(\zeta)$ in the expression for the distribution amplitude $d_{b \bar{b}_{8 T} \rightarrow g}(\zeta)$ in Eq. (32). The expression for $d_{0}(\zeta)$ is obtained by setting $m_{b}=0$ in Eq. (31). The resulting dimensionally regularized integral is 0 because it has no scale. The last term on the right side of Eq. (47) is given by the integral in Eq. (35) with $m_{b}=0$. The three denominators are proportional to $q \cdot \bar{n}, q^{2}$, and $q . n$. This dimensionally regularized integral is also zero because it has no scale. Thus, the only nonzero terms on the right side of Eq. (47) with $m_{b}=0$ are the first and second terms, which are given in Eqs. (50) and (55).

Subtracting Eq. (55) from Eq. (50), we get the regularized $m_{H}$-dependent hard form factor:

$$
\mathcal{F}_{\text {hard }}^{\mathrm{LPb}}\left(\hat{s}, m_{H}^{2}\right)=-\frac{g_{s}^{2} y_{b}}{16 \pi^{2}}\left[\frac{-\hat{s}-i \epsilon}{\mu^{2}}\right]^{-\epsilon}\left(\frac{1}{\epsilon^{2}}+\frac{2}{\epsilon}-\frac{\pi^{2}}{6}+6-\frac{2 m_{H}^{2}}{\hat{s}-m_{H}^{2}} \log \frac{\hat{s}+i \epsilon}{m_{H}^{2}}\right) .
$$

This reduces to the hard form factor in Eq. (16) in the limit $m_{H} \rightarrow 0$. The renormalized $m_{H}$-dependent hard form factor can be obtained by minimal subtraction of the poles in $\epsilon$ :

$$
\tilde{\mathcal{F}}_{H+g}^{(H)}\left(\hat{s}, m_{H}^{2}\right)=\frac{g_{s}^{2} y_{b}}{16 \pi^{2}}\left(-\frac{1}{2} \log ^{2} \frac{-\hat{s}-i \epsilon}{\mu^{2}}+2 \log \frac{-\hat{s}-i \epsilon}{\mu^{2}}+\frac{\pi^{2}}{6}-6+\frac{2 m_{H}^{2}}{\hat{s}-m_{H}^{2}} \log \frac{\hat{s}+i \epsilon}{m_{H}^{2}}\right) .
$$

The explicit form of the $\mathrm{LP} b$ factorization formula in Eq. (45), which includes all terms at a leading power of $m_{b}^{2} / Q^{2}$, is

$$
\begin{aligned}
\mathcal{F}^{\mathrm{LP} b}\left(\hat{s}, m_{b}^{2}, m_{H}^{2}\right) \equiv & \tilde{\mathcal{F}}_{H+g}^{(H)}\left(\hat{s}, m_{H}^{2}\right)+\int_{-1}^{+1} d \zeta \tilde{\mathcal{F}}_{b \bar{b}_{1 V}+g}(\zeta) d_{b \bar{b}_{1 V} \rightarrow H}\left(\zeta ; m_{b}^{2}, m_{H}^{2}, P . n\right)+\int_{-1}^{+1} d \zeta \tilde{\mathcal{F}}_{H+b \bar{b}_{8 T}}(\zeta) d_{b \bar{b}_{8 T} \rightarrow g}\left(\zeta ; m_{b}^{2}, p_{3} . \bar{n}\right) \\
& +\mathcal{F}_{\text {endpt }}\left(m_{b}^{2}\right)
\end{aligned}
$$

It differs from the LP form factor in Eq. (6) only by the difference between the $m_{H}$-dependent hard form factor in Eq. (57) and the hard form factor in Eq. (41):

$$
\mathcal{F}^{\mathrm{LP} b}\left(\hat{s}, m_{b}^{2}, m_{H}^{2}\right)=\mathcal{F}^{\mathrm{LP}}\left(\hat{s}, m_{b}^{2}, m_{H}^{2}\right)+\frac{g_{s}^{2} y_{b}}{32 \pi^{2}}\left\{\frac{4 m_{H}^{2}}{\hat{s}-m_{H}^{2}} \log \frac{\hat{s}+i \epsilon}{m_{H}^{2}}\right\} .
$$

\section{COMPARISON WITH FULL FORM FACTOR}

We proceed to compare our approximations to the form factor at LO for $q \bar{q} \rightarrow H+g$ from the bottom-quark loop. The full form factor $\mathcal{F}\left(\hat{s}, m_{b}^{2}, m_{H}^{2}\right)$ is given in Refs. [5,6]. The three approximations are

(i) the LP form factor $\mathcal{F}^{\mathrm{LP}}\left(\hat{s}, m_{b}^{2}, m_{H}^{2}\right)$ in Eq. (6), which is leading power in $m_{H}^{2} / \hat{s}$ and $m_{b}^{2} / \hat{s}$,

(ii) the $\mathrm{LP} b$ form factor $\mathcal{F}^{\mathrm{LP} b}\left(\hat{s}, m_{b}^{2}, m_{H}^{2}\right)$ in Eq. (59), which is leading power in $m_{b}^{2} / \hat{s}$ only, and

(iii) the $m_{b} \rightarrow 0$ form factor $\mathcal{F}\left(\hat{s}, m_{b}^{2} \rightarrow 0, m_{H}^{2}\right)$ in Eq. (8), which is obtained from the full form factor by taking the limit $m_{b} \rightarrow 0$ except in logarithms of $m_{b}$.

The full form factor and the approximations depend on the coupling constants $g_{s}$ and $y_{b}=m_{b} / v$ and on the masses
$m_{H}$ and $m_{b}$. The mass of the Higgs is $m_{H}=125 \mathrm{GeV}$. The running bottom-quark mass at the scale of the Higgs mass is $m_{b}\left(m_{H}\right)=3.06 \mathrm{GeV}$.

The form factor $\mathcal{F}$ at LO has an overall coupling constant factor $g_{s}^{2} y_{b}$. The coupling constants depend logarithmically on momentum scales that can range from $m_{b}$ to $m_{H}$ and to $\sqrt{\hat{s}}$. One advantage of the LP factorization formula is that the separation of scales allows the momentum scales of the coupling constants to be determined. In the hard form factor in Eq. (41), the appropriate scale is $\sqrt{\hat{s}}$, so the coupling constant factor is $g_{s}^{2}(\sqrt{\hat{s}}) y_{b}(\sqrt{\hat{s}})$. In the Higgs collinear contribution, the coupling constant factor in the hard form factor for $g^{*} \rightarrow b \bar{b}_{1 V}+g$ in Eq. (19) is $g_{s}^{2}(\sqrt{\hat{s}})$, and the Yukawa coupling constant in the distribution amplitude for $b \bar{b}_{1 V} \rightarrow H$ in Eq. (42) is $y_{b}\left(m_{H}\right)$. In the gluon collinear 


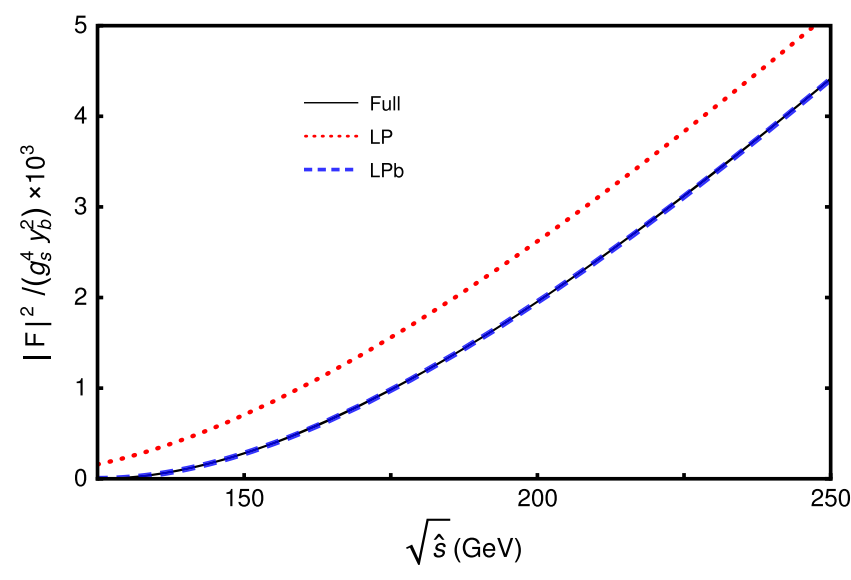

FIG. 6. Form factors for $q \bar{q} \rightarrow H+g$ with a bottom-quark loop as functions of the center-of-mass energy $\sqrt{\hat{s}}$ : the full form factor $|\mathcal{F}|^{2}$ (solid curve), the LP form factor (dotted curve), and the LP $b$ form factor (dashed curve). The $m_{b} \rightarrow 0$ form factor is indistinguishable from the full form factor in this plot.

contribution, the coupling constant factor in the hard form factor for $g^{*} \rightarrow H+b \bar{b}_{8 T}$ in Eq. (30) is $g_{s}(\sqrt{\hat{s}}) y_{b}(\sqrt{\hat{s}})$, and the coupling constant in the distribution amplitude for $b \bar{b}_{8 T} \rightarrow g$ in Eq. (43) is $g_{s}\left(m_{b}\right)$. In the end point contribution in Eq. (44), the coupling constant in the hard amplitude for producing $b+\bar{b}$ is $g_{s}(\sqrt{\hat{s}})$, and the coupling constant factor in the soft amplitude for the transition $b \bar{b} \rightarrow$ $H+g$ is $g_{s}\left(m_{b}\right) y_{b}\left(m_{H}\right)$. Replacing the coupling constants by the appropriate running coupling constants resums some leading logarithms to all orders. However, there is little to be gained by this partial resummation unless all the leading logarithms are summed to all orders. If we choose the common scale $m_{H}$ for all the coupling constants, the coupling constant factor $g_{s}^{4} y_{b}^{2}$ in $|\mathcal{F}|^{2}$ is approximately $2.4 \times 10^{-4}$. We will compare the approximations to $|\mathcal{F}|^{2}$ in ways that do not depend on the coupling constant factor.

In Fig. 6, the absolute squares of the form factors divided by the coupling constant factor $g_{s}^{4} y_{b}^{2}$ are shown as functions of the center-of-mass energy $\sqrt{\hat{s}}$, which ranges from the threshold $m_{H}$ for producing the Higgs to $250 \mathrm{GeV}$. The $m_{b} \rightarrow 0$ form factor is indistinguishable from the full form factor in this plot. The $\mathrm{LP} b$ form factor is almost indistinguishable from the full form factor. The error in the LP form factor does not seem to be decreasing as $\hat{s}$ increases.

In Fig. 7, we compare the percentage errors in the three approximations to $|\mathcal{F}|^{2}$. The percentage error is defined as the difference from $|\mathcal{F}|^{2}$ divided by $|\mathcal{F}|^{2}$. In the left panel of Fig. 7, which is linear in $\sqrt{\hat{s}}$ out to $10 m_{H}$, all three approximations appear to be converging to $|\mathcal{F}|^{2}$ as $\hat{s}$ increases but with very different rates of convergence. The percentage error in the LP form factor approaches 0 from above, decreasing to less than $5 \%$ for $\sqrt{\hat{s}}$ greater than about $400 \mathrm{GeV}$. The $\mathrm{LP} b$ form factor is a much better approximation. The percentage error approaches 0 from below, and it is already less than $5 \%$ at $\sqrt{\hat{s}}=129 \mathrm{GeV}$. The $m_{b} \rightarrow 0$ form factor seems to be a much better approximation than the $\mathrm{LP} b$ form factor. The percentage error seems to be approaching 0 from above, and it is always less than $0.08 \%$. In the right panel of Fig. 7, which is logarithmic in $\sqrt{\hat{s}}$ from $10 m_{H}$ to $100 m_{H}$, all three approximations seem to continue converging to $|\mathcal{F}|^{2}$.
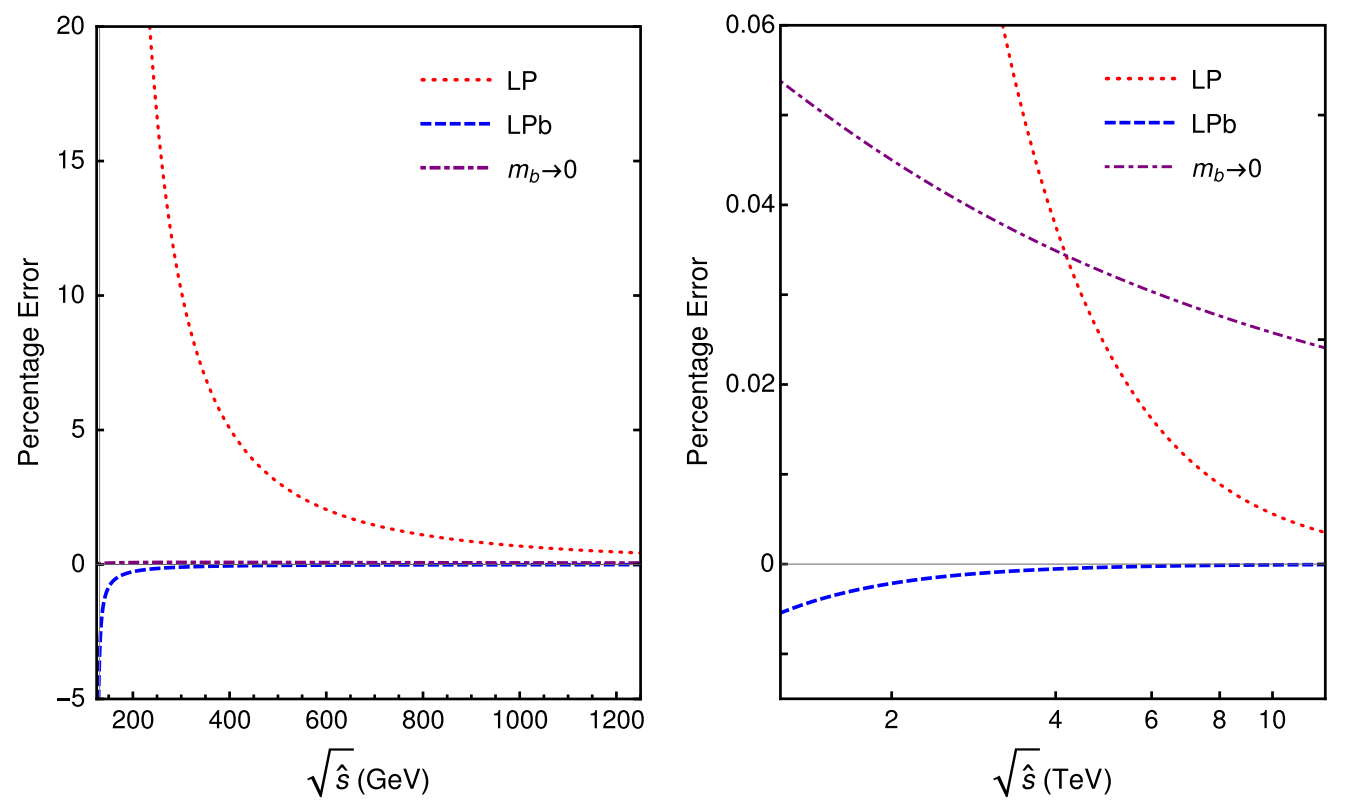

FIG. 7. Percentage errors in form factors for $q \bar{q} \rightarrow H+g$ with a bottom-quark loop as functions of the center-of-mass energy $\sqrt{\hat{s}}$ : the LP form factor (dotted curve), the LP $b$ form factor (dashed curve), and the $m_{b} \rightarrow 0$ form factor (dot-dashed curve). The ranges of $\sqrt{\hat{s}}$ are from $m_{H}$ to $10 m_{H}$ on a linear scale (left panel) and from $10 m_{H}$ to $100 m_{H}$ on a log scale (right panel). 
However, the rates of convergence of the $\mathrm{LP} b$ form factor and the LP form factor are much more rapid, consistent with errors that scale as $1 / \hat{s}$. The $\mathrm{LP} b$ form factor is a better approximation than the $m_{b} \rightarrow 0$ form factor for $\sqrt{\hat{s}}$ greater than about $330 \mathrm{GeV}$. The LP form factor is a better approximation than the $m_{b} \rightarrow 0$ form factor for $\sqrt{\hat{s}}$ greater than about $4.1 \mathrm{TeV}$. The error in the $m_{b} \rightarrow 0$ form factor actually approaches a nonzero constant at large $\hat{s}$. The leading term in the expansion of the error in powers of $m_{b}^{2} / m_{H}^{2}$ is

$$
\begin{gathered}
\mathcal{F}\left(\hat{s}, m_{b}^{2} \rightarrow 0, m_{H}^{2}\right)-\mathcal{F}\left(\hat{s}, m_{b}^{2}, m_{H}^{2}\right) \\
\rightarrow \frac{g_{s}^{2} y_{b} m_{b}^{2}}{8 \pi^{2} m_{H}^{2}}\left(-\log \frac{m_{H}^{2}}{m_{b}^{2}}-2+i \pi\right) .
\end{gathered}
$$

The error is small because it is suppressed by a factor of $m_{b}^{2} / m_{H}^{2}$. In the right panel of Fig. 7, the decrease in the percentage error for the $m_{b} \rightarrow 0$ form factor as $\hat{s}$ increases is due to the denominator $|\mathcal{F}|^{2}$ increasing as $\log ^{4}\left(\hat{s} / m_{H}^{2}\right)$.

\section{SUMMARY AND OUTLOOK}

In this work, we applied factorization methods developed for QCD to the amplitude for Higgs production at large transverse momentum through a bottom-quark loop. That amplitude is complicated by multiple energy scales: the hard kinematic scales $Q \sim P_{T}, \hat{s}^{1 / 2}$ and the soft mass scales $M \sim m_{b}, m_{H}$. Factorization can be used to separate the scales $M$ and $Q$ and expand the amplitude in powers of $M^{2} / Q^{2}$. To illustrate the factorization approach, we applied it to the bottom-quark-loop contribution to the amplitude for the parton process $q \bar{q} \rightarrow H+g$ at LO in $\alpha_{s}$. The matrix element for this parton process is determined by the form factor $\mathcal{F}\left(\hat{s}, m_{b}^{2}, m_{H}^{2}\right)$ defined in Eq. (3). We defined the leading-power form factor $\mathcal{F}^{\mathrm{LP}}$ to be the leading terms in the expansion of $\mathcal{F}$ in powers of $M^{2} / Q^{2}$. A factorization formula for the LP form factor in which the scales $Q$ and $M$ are separated is given schematically in Eq. (11). The explicit renormalized form of the factorization formula is given in Eq. (40). Each piece in the LP factorization formula was obtained through a diagrammatic calculation that involves fewer scales than those in the calculation of the full form factor. We also defined the $\mathrm{LP} b$ form factor $\mathcal{F}^{\mathrm{LP} b}$ to be the leading terms in the expansion of $\mathcal{F}$ in powers of $m_{b}^{2} / \hat{s}$, keeping all dependence on $m_{H}$ that is not suppressed by $m_{b}^{2} / \hat{s}$. The $\mathrm{LP} b$ form factor is defined by the factorization formula in Eq. (58), and it is given expliicitly in Eq. (59).

In the regularized form of the factorization formula for the LP form factor given schematically in Eq. (11), each of the four terms comes from a different region of the loop momentum of the $b$ quark. The method of regions introduces rapidity divergences in addition to the infrared and ultraviolet divergences that are regularized by dimensional regularization in $4-2 \epsilon$ dimensions. We regularized the rapidity divergences using rapidity regularization with regularization parameter $\eta$. The poles in $\eta$ cancel when the last three terms in the factorization formula in Eq. (11) are added. The poles in $\epsilon$ also cancel when all four terms in the factorization formula are added, provided the rapidity regularization scales satisfy the constraint in Eq. (39). It would be preferable to deduce this constraint from deeper theoretical considerations. With rapidity regularization and zero-bin subtraction, the poles in $\eta$ are ultraviolet divergences. The minimal subtraction of the poles in $\eta$ and in $\epsilon$ in each of the regularized pieces of the factorization formula can therefore be interpreted as a renormalization procedure.

In the renormalized factorization formula for the LP form factor in Eq. (40), the hard scales $Q$ and the soft scales $M$ are separated. The hard contribution depends only on the hard scale $Q$, and it is given in Eq. (41). The Higgs collinear and gluon collinear contributions were each expressed as an integral over the relative longitudinal momentum fraction $\zeta$ of a $b$ quark of the product of a form factor that depends on the hard scale $Q$ and a distribution amplitude that depends on the soft scale $M$. The integrand for the Higgs collinear contribution is the product of the hard form factor for $t \bar{t}_{1 V}+g$ in Eq. (19) and the distribution amplitude for $t \bar{t}_{1 V}$ in the Higgs in Eq. (42). The integrand for the gluon collinear contribution is the product of the hard form factor for $H+t \bar{t}_{8 T}$ in Eq. (30) and the distribution amplitude for $t \bar{t}_{8 T}$ in a real gluon in Eq. (43). The end point contribution to the LP form factor depends only on the scale $M$, and it is given in Eq. (44).

The LP form factor $\mathcal{F}^{\mathrm{LP}}$ is a good approximation to the full form factor only at extremely large $\hat{s}$. The error is of order $m_{H}^{2} / \hat{s}$, so the error decreases to 0 as $\hat{s}$ increases. As shown in Fig. 7, the percentage error in $\left|\mathcal{F}^{\mathrm{LP}}\right|^{2}$ does not decrease to less than $5 \%$ until $\sqrt{\hat{s}}>400 \mathrm{GeV}$. Thus, the LP form factor has no practical use at LHC energies. We defined the $\mathrm{LP} b$ form factor $\mathcal{F}^{\mathrm{LP} b}$ by the simple modification of the factorization formula in Eq. (58). It differs from the LP form factor only in the $m_{H}$-dependent hard form factor, which is given in Eq. (59). This hard form factor can be obtained from additional calculations with $m_{b}=0$. The error in the $\mathrm{LP} b$ form factor is order $m_{b}^{2} / \hat{s}$. As shown in Fig. 7, the percentage error in $\left|\mathcal{F}^{\mathrm{LP} b}\right|^{2}$ is already less than $5 \%$ at $\sqrt{\hat{s}}=129 \mathrm{GeV}$.

Our factorization formula for the form factor $\mathcal{F}$ for the parton process $q \bar{q} \rightarrow H+g$ is the sum of four terms: a hard term, two collinear terms, and an end point term. It can be adapted to the bottom-quark-loop contributions to the form factors for the parton processes $g q \rightarrow H+q$ and $g \bar{q} \rightarrow$ $H+\bar{q}$ by analytically continuing the positive Mandelstam variable $\hat{s}$ to a negative Mandelstam variable $\hat{t}$. The factorization formula for those form factors involves a distribution amplitude for $b \bar{b}$ in an incoming gluon instead of the distribution amplitude for $b \bar{b}$ in an outgoing gluon. 
The factorization formula for the bottom-quark-loop contribution for the parton process $g g \rightarrow H+g$ at $\mathrm{LO}$ can be derived using similar methods, but it is more complicated. It has a hard term, four collinear terms, and perhaps as many as six end point terms. All the pieces in the factorization formula can be obtained directly from Feynman diagrams by calculations that involve fewer scales than the full matrix element.

Our factorization approach can be extended to NLO in $\alpha_{s}$. The NLO calculation of the form factor for $q \bar{q} \rightarrow H+g$ would require calculating each of the pieces in the factorization formula in Eq. (40) to NLO. The NLO calculations of the hard form factors $\tilde{\mathcal{F}}_{H+g}, \tilde{\mathcal{F}}_{b \bar{b}_{1 V}+g}$, and $\tilde{\mathcal{F}}_{H+b \bar{b}_{8 T}}$ require straightforward perturbative QCD calculations with massless quarks and massless Higgs. The NLO calculation of the end point form factor $\mathcal{F}_{\text {endpt }}$ may also require factorization since it may have nontrivial dependence on the scale $Q$ through the hard form factor $\tilde{\mathcal{F}}_{b+\bar{b}}$ for producing $b+\bar{b}$. The NLO calculation of the fragmentation amplitudes for $b \bar{b}_{1 V} \rightarrow H$ and for $b \bar{b}_{8 T} \rightarrow g$ may be the most challenging step in the NLO calculation of the LP form factor. At NLO, there may be additional terms in the factorization formula associated with other double-parton channels, such as $b \bar{b}_{1 S}, b \bar{b}_{1 T}, b \bar{b}_{8 S}$, and $b \bar{b}_{8 V}$. These additional terms would require only LO calculations.

Our factorization formula could be derived more formally using effective field theory methods analogous to those used in soft collinear effective field theory in QCD. The individual pieces in the factorization formula could all be expressed in terms of matrix elements of operators in the effective field theory. These formal definitions could be useful in the calculation of the form factor to higher orders in $\alpha_{s}$. They would also facilitate the all-order resummation of large logarithms of $P_{T}^{2} / m_{b}^{2}$ by solving renormalization group equations.

Our factorization formula can also be extended to study the interference of top-quark-loop diagrams with bottom-quark-loop diagrams for Higgs production. For the interference contribution relevant to the LHC phenomenology, it is most convenient to use HEFT for the diagrams with a top-quark loop and to use our $\mathrm{LP} b$ formalism for the diagrams with a bottom-quark loop. The top-quark loop amplitude involves only the amplitude for direct Higgs production. The bottom-quark loop amplitude involves the amplitude for direct Higgs production as well as $b \bar{b}$-toHiggs distribution amplitudes. The all-orders proof of factorization and the derivation of the evolution equations for Higgs production are straightforward generalizations of those for the factorization of power corrections in QCD [54].

We expect the $\mathrm{LP} b$ factorization formula at NLO to be useful phenomenologically at the LHC. The effect of the bottom-quark mass on the Higgs $P_{T}$ distribution at the LHC is expected to be at most $-8 \%$ for $P_{T}<50 \mathrm{GeV}$ [55]. The fractional error of the $\mathrm{LP} b$ factorization formula at $\mathrm{LO}$ is order $m_{b}^{2} / Q^{2}$. If the leading logarithms of $P_{T} / m_{b}$ can be resummed to all orders, the fractional error of the $\mathrm{LP} b$ factorization formula at NLO is order $\alpha_{s} m_{b}^{2} / Q^{2}$. This error may be sufficient for phenomenological purposes at the LHC.

\section{ACKNOWLEDGMENTS}

This work of E. B. and H. Z. was supported in part by the Department of Energy under Award No. DE-SC0011726. J.W.Z. was supported in part by the Natural Science Foundation of China under Grant No. 11347024, the Natural Science Foundation Project of CQCSTC under Grants No. 2014jcyjA00030 and No. 2016jcyjA0336, the Scientific and Technological Research Program of Chongqing Municipal Education Commission under Grant No. KJ1401313, and the Research Foundation of Chongqing University of Science and Technology under Grant No. CK2016Z03. We acknowledge the use of FeynCalc $[56,57]$ in this research.
[1] G. Aad et al. (ATLAS Collaboration), Observation of a new particle in the search for the Standard Model Higgs boson with the ATLAS detector at the LHC, Phys. Lett. B 716, 1 (2012).

[2] S. Chatrchyan et al. (CMS Collaboration), Observation of a new boson at a mass of $125 \mathrm{GeV}$ with the CMS experiment at the LHC, Phys. Lett. B 716, 30 (2012).

[3] D. de Florian et al. (LHC Higgs Cross Section Working Group), Handbook of LHC Higgs cross sections: 4. Deciphering the nature of the Higgs sector, CERN Yellow Reports: Monographs (2017), Vol. 2.
[4] J. M. Lindert, K. Melnikov, L. Tancredi, and C. Wever, Top-Bottom Interference Effects in Higgs Plus Jet Production at the LHC, Phys. Rev. Lett. 118, 252002 (2017).

[5] R. K. Ellis, I. Hinchliffe, M. Soldate, and J. J. van der Bij, Higgs decay to $\tau^{+} \tau^{-}$: a possible signature of intermediate mass Higgs bosons at the SSC, Nucl. Phys. B297, 221 (1988).

[6] U. Baur and E. W. N. Glover, Higgs boson production at large transverse momentum in hadronic collisions, Nucl. Phys. B339, 38 (1990). 
[7] R. Mueller and D. G. Öztürk, On the computation of finite bottom-quark mass effects in Higgs boson production, J. High Energy Phys. 08 (2016) 055.

[8] K. Melnikov, L. Tancredi, and C. Wever, Two-loop $g g \rightarrow$ $\mathrm{Hg}$ amplitude mediated by a nearly massless quark, J. High Energy Phys. 11 (2016) 104.

[9] K. Melnikov, L. Tancredi, and C. Wever, Two-loop amplitudes for $q g \rightarrow H q$ and $q \bar{q} \rightarrow H g$ mediated by a nearly massless quark, Phys. Rev. D 95, 054012 (2017).

[10] G. Bozzi, S. Catani, D. de Florian, and M. Grazzini, The $q_{T}$ spectrum of the Higgs boson at the LHC in QCD perturbation theory, Phys. Lett. B 564, 65 (2003).

[11] A. Kulesza, G. F. Sterman, and W. Vogelsang, Joint resummation for Higgs production, Phys. Rev. D 69, 014012 (2004).

[12] G. Bozzi, S. Catani, D. de Florian, and M. Grazzini, Transverse-momentum resummation and the spectrum of the Higgs boson at the LHC, Nucl. Phys. B737, 73 (2006).

[13] S. Catani and M. Grazzini, Higgs boson production at hadron colliders: hard-collinear coefficients at the NNLO, Eur. Phys. J. C 72, 2013 (2012); Erratum,Eur. Phys. J. C 72, 2132 (2012).

[14] T. Becher, M. Neubert, and D. Wilhelm, Higgs-boson production at small transverse momentum, J. High Energy Phys. 05 (2013) 110.

[15] D. Neill, I. Z. Rothstein, and V. Vaidya, The higgs transverse momentum distribution at NNLL and its theoretical errors, J. High Energy Phys. 12 (2015) 097.

[16] S. Forte and C. Muselli, High energy resummation of transverse momentum distributions: Higgs in gluon fusion, J. High Energy Phys. 03 (2016) 122.

[17] P. F. Monni, E. Re, and P. Torrielli, Higgs TransverseMomentum Resummation in Direct Space, Phys. Rev. Lett. 116, 242001 (2016).

[18] F. Caola, S. Forte, S. Marzani, C. Muselli, and G. Vita, The Higgs transverse momentum spectrum with finite quark masses beyond leading order, J. High Energy Phys. 08 (2016) 150.

[19] C. Muselli, S. Forte, and G. Ridolfi, Combined threshold and transverse momentum resummation for inclusive observables, J. High Energy Phys. 03 (2017) 106.

[20] W. Bizon, P. F. Monni, E. Re, L. Rottoli, and P. Torrielli, Momentum-space resummation for transverse observables and the Higgs $p_{\perp}$ at $\mathrm{N}^{3} \mathrm{LL}+\mathrm{NNLO}$, J. High Energy Phys. 02 (2018) 108.

[21] H. Mantler and M. Wiesemann, Top- and bottom-mass effects in hadronic Higgs production at small transverse momenta through LO + NLL, Eur. Phys. J. C 73, 2467 (2013).

[22] M. Grazzini and H. Sargsyan, Heavy-quark mass effects in Higgs boson production at the LHC, J. High Energy Phys. 09 (2013) 129.

[23] A. Banfi, P. F. Monni, and G. Zanderighi, Quark masses in Higgs production with a jet veto, J. High Energy Phys. 01 (2014) 097.

[24] E. Bagnaschi, R. V. Harlander, H. Mantler, A. Vicini, and M. Wiesemann, Resummation ambiguities in the Higgs transverse-momentum spectrum in the Standard Model and beyond, J. High Energy Phys. 01 (2016) 090.
[25] K. Melnikov and A. Penin, On the light quark mass effects in Higgs boson production in gluon fusion, J. High Energy Phys. 05 (2016) 172.

[26] C. Anastasiou, C. Duhr, F. Dulat, F. Herzog, and B. Mistlberger, Higgs Boson Gluon-Fusion Production in QCD at Three Loops, Phys. Rev. Lett. 114, 212001 (2015).

[27] C. Anastasiou, C. Duhr, F. Dulat, E. Furlan, T. Gehrmann, F. Herzog, A. Lazopoulos, and B. Mistlberger, High precision determination of the gluon fusion Higgs boson cross-section at the LHC, J. High Energy Phys. 05 (2016) 058.

[28] V. Ahrens, T. Becher, M. Neubert, and L. L. Yang, Renormalization-group improved prediction for Higgs production at hadron colliders, Eur. Phys. J. C 62, 333 (2009).

[29] M. Bonvini and S. Marzani, Resummed Higgs cross section at $N^{3}$ LL, J. High Energy Phys. 09 (2014) 007.

[30] Y. Li, A. von Manteuffel, R. M. Schabinger, and H. X. Zhu, Soft-virtual corrections to Higgs production at $\mathrm{N}^{3} \mathrm{LO}$, Phys. Rev. D 91, 036008 (2015).

[31] M. Bonvini and L. Rottoli, Three loop soft function for $\mathrm{N}^{3} \mathrm{LL}$ gluon fusion Higgs production in soft-collinear effective theory, Phys. Rev. D 91, 051301 (2015).

[32] T. Schmidt and M. Spira, Higgs boson production via gluon fusion: soft-gluon resummation including mass effects, Phys. Rev. D 93, 014022 (2016).

[33] M. Bonvini, S. Marzani, C. Muselli, and L. Rottoli, On the Higgs cross section at $\mathrm{N}^{3} \mathrm{LO}+\mathrm{N}^{3} \mathrm{LL}$ and its uncertainty, $\mathrm{J}$. High Energy Phys. 08 (2016) 105.

[34] R. Boughezal, F. Caola, K. Melnikov, F. Petriello, and M. Schulze, Higgs boson production in association with a jet at next-to-next-to-leading order in perturbative QCD, J. High Energy Phys. 06 (2013) 072.

[35] X. Chen, T. Gehrmann, E. W. N. Glover, and M. Jaquier, Precise QCD predictions for the production of Higgs + jet final states, Phys. Lett. B 740, 147 (2015).

[36] R. Boughezal, F. Caola, K. Melnikov, F. Petriello, and M. Schulze, Higgs Boson Production in Association with a Jet at Next-to-Next-to-Leading Order, Phys. Rev. Lett. 115, 082003 (2015).

[37] R. Boughezal, C. Focke, W. Giele, X. Liu, and F. Petriello, Higgs boson production in association with a jet at NNLO using jettiness subtraction, Phys. Lett. B 748, 5 (2015).

[38] J. M. Campbell, R. K. Ellis, and G. Zanderighi, Next-toleading order Higgs +2 jet production via gluon fusion, J. High Energy Phys. 10 (2006) 028.

[39] J. M. Campbell, R. K. Ellis, and C. Williams, Hadronic production of a Higgs boson and two jets at next-to-leading order, Phys. Rev. D 81, 074023 (2010).

[40] G. Cullen, H. van Deurzen, N. Greiner, G. Luisoni, P. Mastrolia, E. Mirabella, G. Ossola, T. Peraro, and F. Tramontano, NLO QCD Corrections to Higgs Boson Production Plus Three Jets in Gluon Fusion, Phys. Rev. Lett. 111 (2013) 131801.

[41] E. Braaten and H. Zhang, Inclusive Higgs production at large transverse momentum, Phys. Rev. D 93, 053014 (2016).

[42] E. Braaten, H. Zhang, and J. W. Zhang, Mass dependence of Higgs production at large transverse momentum, J. High Energy Phys. 11 (2017) 127. 
[43] Z. B. Kang, J. W. Qiu, and G. Sterman, Heavy Quarkonium Production and Polarization, Phys. Rev. Lett. 108, 102002 (2012).

[44] S. Fleming, A. K. Leibovich, T. Mehen, and I. Z. Rothstein, The systematics of quarkonium production at the LHC and double parton fragmentation, Phys. Rev. D 86, 094012 (2012).

[45] T. Gehrmann, M. Jaquier, E. W. N. Glover, and A. Koukoutsakis, Two-loop QCD corrections to the helicity amplitudes for $H \rightarrow 3$ partons, J. High Energy Phys. 02 (2012) 056.

[46] W. B. Kilgore, One-loop single-real-emission contributions to $p p \rightarrow H+X$ at next-to-next-to-next-to-leading order, Phys. Rev. D 89, 073008 (2014).

[47] S. Dawson, I. M. Lewis, and M. Zeng, Effective field theory for Higgs boson plus jet production, Phys. Rev. D 90, 093007 (2014).

[48] M. Beneke and V. A. Smirnov, Asymptotic expansion of Feynman integrals near threshold, Nucl. Phys. B522, 321 (1998).

[49] V. A. Smirnov, Applied asymptotic expansions in momenta and masses, Springer Tracts Mod. Phys. 177, 1 (2002).

[50] A. V. Manohar and I. W. Stewart, The zero-bin and mode factorization in quantum field theory, Phys. Rev. D 76, 074002 (2007).
[51] J. Y. Chiu, A. Jain, D. Neill, and I. Z. Rothstein, The Rapidity Renormalization Group, Phys. Rev. Lett. 108, 151601 (2012).

[52] J. Y. Chiu, A. Jain, D. Neill, and I. Z. Rothstein, A formalism for the systematic treatment of rapidity logarithms in quantum field theory, J. High Energy Phys. 05 (2012) 084.

[53] Y. Q. Ma, J. W. Qiu, and H. Zhang, Heavy quarkonium fragmentation functions from a heavy quark pair. I. $S$ wave, Phys. Rev. D 89, 094029 (2014).

[54] J. W. Qiu and G. Sterman, Power corrections to hadronic scattering. 2. Factorization, Nucl. Phys. B353, 137 (1991).

[55] W. Y. Keung and F. J. Petriello, Electroweak and finite quark-mass effects on the Higgs boson transverse momentum distribution, Phys. Rev. D 80, 013007 (2009).

[56] R. Mertig, M. Bohm, and A. Denner, FEYNCALC: Computer algebraic calculation of Feynman amplitudes, Comput. Phys. Commun. 64, 345 (1991).

[57] V. Shtabovenko, R. Mertig, and F. Orellana, New developments in FeynCalc 9.0, Comput. Phys. Commun. 207, 432 (2016). 